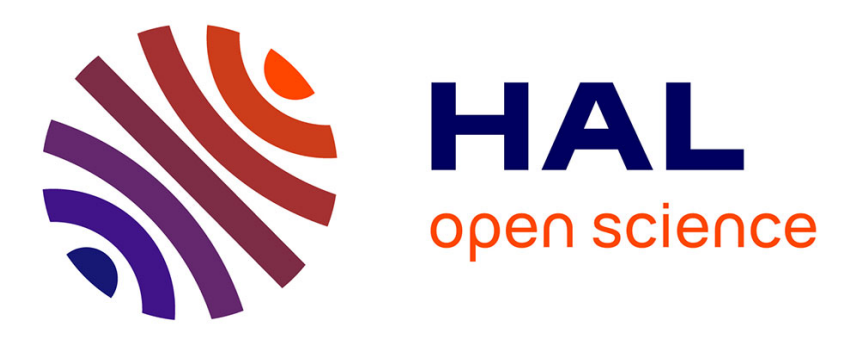

\title{
Three-dimensional effect of high frequency vibration on convection in silicon melt
}

Faiza Mokhtari, Slim Kaddeche, Daniel Henry, Samia Bouarab, Abdessamed Medelfef, Valéry Botton

\section{- To cite this version:}

Faiza Mokhtari, Slim Kaddeche, Daniel Henry, Samia Bouarab, Abdessamed Medelfef, et al.. Threedimensional effect of high frequency vibration on convection in silicon melt. Physical Review Fluids, 2020, 5 (12), 10.1103/PhysRevFluids.5.123501 . hal-03065529

\section{HAL Id: hal-03065529 \\ https://hal.science/hal-03065529}

Submitted on 14 Dec 2020

HAL is a multi-disciplinary open access archive for the deposit and dissemination of scientific research documents, whether they are published or not. The documents may come from teaching and research institutions in France or abroad, or from public or private research centers.
L'archive ouverte pluridisciplinaire HAL, est destinée au dépôt et à la diffusion de documents scientifiques de niveau recherche, publiés ou non, émanant des établissements d'enseignement et de recherche français ou étrangers, des laboratoires publics ou privés. 


\title{
Three-dimensional effect of high frequency vibration on convection in silicon melt
}

\author{
Faiza Mokhtari $\odot,{ }^{1}$ Slim Kaddeche $\odot,{ }^{2}$ Daniel Henry $\odot,{ }^{3}$ Samia Bouarab,${ }^{1,4}$ \\ Abdessamed Medelfef $\odot{ }^{1}{ }^{1}$ and Valéry Botton $\oplus^{3,5}$ \\ ${ }^{1}$ Laboratoire de Thermodynamique et Systèmes Energétiques, Faculté de Physique, Université des Sciences \\ et de la Technologie Houari Boumediène, BP 32, 16111 Bab Ezzouar, Alger, Algeria \\ ${ }^{2}$ Laboratoire de Recherche Matériaux, Mesures et Applications LR-11-ES-25, Institut National \\ des Sciences Appliquées et de Technologie, BP 676, 1080 Tunis Cedex, Tunisia \\ ${ }^{3}$ Laboratoire de Mécanique des Fluides et d'Acoustique, CNRS, Université de Lyon, Ecole Centrale \\ de Lyon/Université Lyon 1/INSA Lyon, ECL, 36 Avenue Guy de Collongue, 69134 Ecully Cedex, France \\ ${ }^{4}$ Université M'Hammed Bougara, Boumerdes, Algeria \\ ${ }^{5}$ INSA Euro-Méditerranée, Université Euro-Méditerranéenne de Fès, Route de Meknès, BP51, Fez, Morocco
}

(Received 15 July 2020; accepted 10 November 2020; published 14 December 2020)

\begin{abstract}
Using the Chebyshev spectral method, the effect of high frequency (HF) vibrations on a cubic cell heated from the side and containing a liquid metal is investigated. This study extends the numerical and theoretical two-dimensional results presented in the authors' past work [Phys. Fluids 31, 043605 (2019)] about the influence of HF vibration direction on the flow structure in a rectangular crucible filled with a liquid metal. The vibration direction can now be three dimensional, and not limited to the main flow plane. In practice, the study considers that the vibration vector is contained in one of the three principal planes of the cavity ( $x z, x y$, and $y z$ planes). Two different cases, i.e., under weightlessness and gravity conditions, are considered for each type of vibration to better understand the separate effect of both vibration and buoyancy forces and also their combined effects. Each type of vibration has its own features and affects the flow intensity and patterns differently.
\end{abstract}

DOI: 10.1103/PhysRevFluids.5.123501

\section{INTRODUCTION}

Buoyant flows of liquid metals are omnipresent in many engineering and technological applications such as "crystal growth from the melt" techniques [1]. Among these techniques, we can mention the Czochralski (CZ), the Kyropoulos (KY), the horizontal and vertical Bridgman (HB and VB), and the Floating zone (FZ) techniques. During the solidification process, such flows can have significant effects on the quality of the grown crystals and therefore have to be controlled [2-6].

Several strategies have been proposed to control these buoyant flows. High frequency (HF) vibrations can be used even for not electrically conducting fluids (which is not the case of magnetic fields) and offer promising possibilities to control the flow. It is well known that external vibrations imposed to an enclosure, filled with a fluid subject to temperature gradients, generate thermovibrational convection [7]. To solve such problems, a first possibility is to solve directly the conservation equations, with the vibration modeled as an oscillatory component of gravity, as it is done in [8] for a thermodiffusion process operated under rather low frequency vibration ( $g$ jitters). Nevertheless, if the characteristic time of vibration is far smaller than the other characteristic times, such simulations become excessively expensive. An alternative for small amplitude and high-frequency vibrations is the averaging method [7,9]. As explained in detail by Gershuni and Lyubimov [7], when the vibration period is much smaller than the characteristic hydrodynamic time, the flow field can be decomposed into a fast part and a time-averaged part: the first part oscillates with the high vibration 
frequency and the second part measures the response of the fluid to this vibration, either steady or oscillatory with lower frequencies. The averaging method then allows us to derive a complete set of time-averaged equations governing these time-averaged fields.

The effect of high frequency vibrations has been extensively studied in two-dimensional cavities. An overview of the literature on this topic can be found in [10-19]. It is however crucial to investigate these effects when the problem cannot be modeled through a two-dimensional approach. To our best knowledge, only a few works have explored this domain.

Biringen et al. [20] studied the effect of gravity modulation on three-dimensional RayleighBénard convection for water $(\operatorname{Pr}=7)$ and air $(\operatorname{Pr}=0.71)$. They have found that, for some vibrational parameter combinations and random modulations in the low-gravity environment, local perturbations are generated, which can potentially influence experimental results. Savino et al. [21] have investigated the effect of high frequency vibration on fluid flow contained in a cubic enclosure under the action of an oscillatory acceleration with an orientation along the direction perpendicular to the applied temperature gradient. Their work aimed to design microgravity experiments using silicone oil with high Prandtl number value $(\mathrm{Pr}=10)$. They have found a qualitative agreement with the two-dimensional results obtained by Farooq and Homsy [22]. Shevtsova et al. [23] performed three-dimensional simulations in a cubic cell filled with isopropanol at high Prandtl number value $(\operatorname{Pr}=27.8)$ under microgravity conditions $\left(g=9.81 \times 10^{-2}\right)$ and harmonic oscillations perpendicular to the imposed vertical temperature gradient. The authors found a very good agreement between experimental results in microgravity environment and three-dimensional numerical results using the real microgravity oscillatory profiles recorded by the on-board accelerometer, with similar flow structure characteristics.

Most of the existing studies have considered the effect of only one direction of vibrations. It is, however, interesting to know the response of the three-dimensional buoyant flow to the influence of vibrations with different directions. This can help us to select efficient vibrations that can either stabilize or enhance the fluid motion and to avoid vibrations that disturb the flow without interesting effect.

The main objective of the present work is to investigate the effect of high frequency (HF) vibrations on a silicon buoyant melt flow contained in a cubic enclosure heated from the side and subjected to HF vibrations with different directions. These three-dimensional results will also be helpful to evaluate the range of validity of the two-dimensional assumption usually adopted in previous studies.

The paper is organized as follows: we first describe the mathematical formulation of the fully three-dimensional (3D) model. The 3D simulation results are then validated by comparison with 3D results [21] and with previously published $2 \mathrm{D}$ results for the whole range of vibration directions contained in the main flow plane, i.e., the plane of the 2D approach [18]. The 3D simulations results obtained for the cases where the vibration direction is contained in one of the three principal planes (i.e., the planes perpendicular to the three axes of the cavity) are then presented, first in weightlessness conditions (pure thermovibrational effect) and then in gravity conditions. Special attention is given to the comparison between the effects obtained on the buoyant flow for vibrations contained in the different principal planes. The final section provides some concluding remarks.

\section{MATHEMATICAL FORMULATION}

In this section, we describe the mathematical model used to perform the numerical simulation of the buoyant flow of molten silicon contained in parallelepipedic crucibles heated from the side and submitted to high frequency (HF) vibrations. Note that the model is derived in the general case of a parallelepipedic cavity, but the numerical results, which are presented in Secs. IV and V, are focused on the case of a cubic cavity.

The geometry of the problem is shown in Fig. 1(a). Two aspect ratios can be defined for this cell, $A_{x}=L / H$ and $A_{y}=W / H$, where $L, H$, and $W$ are the length, height, and width of the crucible, respectively. The molten silicon has a small Prandtl number $\operatorname{Pr}=0.01$. A temperature 


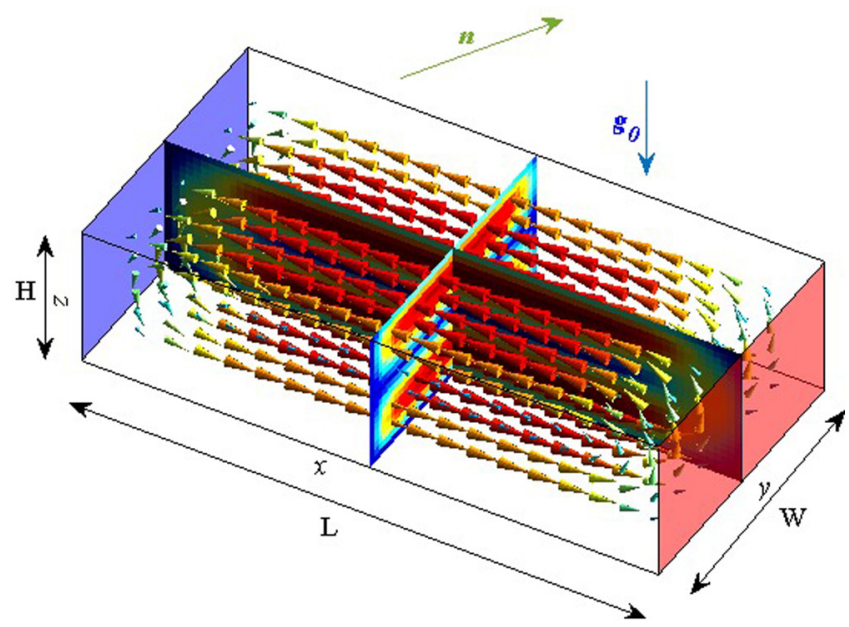

(a)

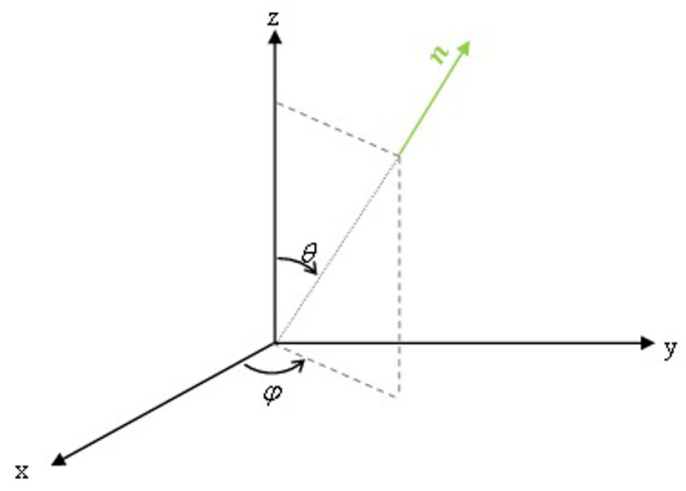

(b)

FIG. 1. (a) The geometry of the problem and the reference buoyant flow in a parallelepipedic cell of length $L$, width $W$, and height $H$. The cavity can be subjected to a high frequency vibration of amplitude $b$ and angular frequency $\Omega . n$ is the unit vector indicating the direction of the vibration and $\boldsymbol{g}_{0}$ is the static gravity acceleration. Hot and cold walls are colored in red and blue, respectively. Contours of velocity magnitude are plotted in the vertical transverse and longitudinal planes. (b) The coordinates system. The direction of vibration given by $\boldsymbol{n}$ is defined with $\theta$ and $\varphi$, the polar and azimuthal angles, respectively.

difference $\Delta T$ is applied between the right and left vertical sidewalls [Fig. 1(a)], which are thermally conducting and maintained at fixed temperatures $T_{h}$ (hot temperature) and $T_{c}$ (cold temperature), respectively, while the other walls are thermally insulated.

The equations governing the melt flow include the effect of both the static gravity acceleration $\boldsymbol{g}_{0}$ and the additional vibrational component $[7,18]$. The resulting acceleration $\boldsymbol{g}$ is then

$$
\boldsymbol{g}=\boldsymbol{g}_{0}+b \Omega^{2} \sin (\Omega t) \boldsymbol{n},
$$

where $b$ is the amplitude of the vibration, $\Omega$ is the angular frequency $(\Omega=2 \pi f$ where $f$ is the frequency), and $\boldsymbol{n}$ is the unit vector indicating the direction of the vibration, which is given by the polar and azimuthal angles $\theta$ and $\varphi$, respectively [Fig. 1(b)]. 
By using the averaging method proposed by Gershuni and Lyubimov [7] in the case of highfrequency harmonic vibrations and using $H, H^{2} / v, v / H, \Delta T / A_{x}=\left(T_{h}-T_{c}\right) / A_{x}$, and $\rho v^{2} / H^{2}$ as scales for length, time, velocity, temperature, and pressure [the temperature is $\left(T-T_{0}\right) A_{x} / \Delta T$, where $T_{0}=\left(T_{h}+T_{c}\right) / 2$ is the mean temperature] as in our previous works [18,19], the dimensionless governing equations for a Newtonian and incompressible buoyant flow under the effect of HF vibrations are

$$
\begin{gathered}
\frac{\partial \boldsymbol{V}}{\partial t}+(\boldsymbol{V} \cdot \nabla) \boldsymbol{V}=-\nabla p+\nabla^{2} \boldsymbol{V}+\mathrm{Gr} T \boldsymbol{\gamma}+\mathrm{Gr}_{\mathrm{v}}(\boldsymbol{W} \cdot \boldsymbol{n}) \nabla T \\
\frac{\partial T}{\partial t}+\boldsymbol{V} \cdot \boldsymbol{\nabla} T=\frac{1}{\operatorname{Pr}} \nabla^{2} T, \\
\nabla \cdot \boldsymbol{V}=0, \\
\nabla \cdot \boldsymbol{W}=0, \\
\nabla \times \boldsymbol{W}=\nabla T \times \boldsymbol{n} .
\end{gathered}
$$

$\boldsymbol{V}$ is the average velocity, often simply called velocity in the following. $\boldsymbol{W}$ is the pulsation velocity, which corresponds to the solenoidal part of the vector field $T \boldsymbol{n}$. The expression of the vibrational force in Eq. (2) comes from that given in Eq. (1.17a) in [7]. The dimensionless parameters, Prandtl number Pr, Grashof number Gr, and vibrational Grashof number $\mathrm{Gr}_{\mathrm{v}}$, are defined respectively as

$$
\operatorname{Pr}=\frac{v}{\chi}, \quad \mathrm{Gr}=\frac{g \beta \Delta T H^{4}}{L v^{2}}, \quad \text { and } \quad \mathrm{Gr}_{\mathrm{v}}=\frac{(b \Omega \beta \Delta T)^{2} H^{4}}{2 L^{2} v^{2}} .
$$

The vector $\boldsymbol{n}$ indicating the direction of vibration [Fig. 1(a)] can be expressed in Cartesian coordinates as a function of the polar $\theta$ and azimuthal $\varphi$ angles [Fig. 1(b)] as

$$
\boldsymbol{n}=\sin \theta \cos \varphi \boldsymbol{i}+\sin \theta \sin \varphi \boldsymbol{j}+\cos \theta \boldsymbol{k} .
$$

Equation (2) and the curl of Eq. (6) [together with Eq. (5)] provide six equations governing velocity and pulsation velocity components $u, v, w$ and $W_{x}, W_{y}, W_{z}$ in $x, y$, and $z$ directions, respectively. These equations can be written as follows:

$$
\begin{gathered}
\frac{\partial u}{\partial t}+u \frac{\partial u}{\partial x}+v \frac{\partial u}{\partial y}+w \frac{\partial u}{\partial z}=-\frac{\partial P}{\partial x}+\mathrm{Gr}_{\mathrm{v}}\left(W_{x} \sin \theta \cos \varphi+W_{y} \sin \theta \sin \varphi+W_{z} \cos \theta\right) \frac{\partial T}{\partial x}+\nabla^{2} u \\
\frac{\partial v}{\partial t}+u \frac{\partial v}{\partial x}+v \frac{\partial v}{\partial y}+w \frac{\partial v}{\partial z}=-\frac{\partial P}{\partial y}+\mathrm{Gr}_{\mathrm{v}}\left(W_{x} \sin \theta \cos \varphi+W_{y} \sin \theta \sin \varphi+W_{z} \cos \theta\right) \frac{\partial T}{\partial y}+\nabla^{2} v \\
\frac{\partial w}{\partial t}+u \frac{\partial w}{\partial x}+v \frac{\partial w}{\partial y}+w \frac{\partial w}{\partial z}=-\frac{\partial P}{\partial z}+\mathrm{Gr}_{\mathrm{v}}\left(W_{x} \sin \theta \cos \varphi+W_{y} \sin \theta \sin \varphi+W_{z} \cos \theta\right) \frac{\partial T}{\partial z} \\
+\mathrm{Gr}^{2}+\nabla^{2} w \\
\nabla^{2} W_{x}=\sin \theta \cos \varphi \nabla^{2} T-\left(\sin \theta \cos \varphi \frac{\partial}{\partial x}+\sin \theta \sin \varphi \frac{\partial}{\partial y}+\cos \theta \frac{\partial}{\partial z}\right) \frac{\partial T}{\partial x} \\
\nabla^{2} W_{y}=\sin \theta \sin \varphi \nabla^{2} T-\left(\sin \theta \cos \varphi \frac{\partial}{\partial x}+\sin \theta \sin \varphi \frac{\partial}{\partial y}+\cos \theta \frac{\partial}{\partial z}\right) \frac{\partial T}{\partial y} \\
\nabla^{2} W_{z}=\cos \theta \nabla^{2} T- \\
\left(\sin \theta \cos \varphi \frac{\partial}{\partial x}+\sin \theta \sin \varphi \frac{\partial}{\partial y}+\cos \theta \frac{\partial}{\partial z}\right) \frac{\partial T}{\partial z}
\end{gathered}
$$




\section{Boundary conditions}

On all the solid walls of the enclosure, no-slip and nonpermeability conditions are imposed for the average velocity $\boldsymbol{V}$ and the pulsation velocity $\boldsymbol{W}$, respectively. As in our previous work [18], the heated walls are the vertical walls at $x=0$ and $x=A_{x}$, which have uniform temperatures $T=-A_{X} / 2$ and $T=A_{X} / 2$, respectively. The remaining walls are thermally insulated.

For the average velocity, the boundary conditions can be written as follows:

$$
u=v=w=0 \quad \text { at } \quad x=0, \quad x=A_{x}, \quad y=0, \quad y=A_{y}, \quad z=0, \quad \text { and } \quad z=1 .
$$

Taking into account Eq. (6), the nonpermeability conditions for the pulsation velocity can be derived. We obtain

$$
\begin{aligned}
W_{x} & =0 \quad \text { at } \quad x=0 \quad \text { and } x=A_{x}, \\
\frac{\partial W_{x}}{\partial y} & =-\frac{\partial T}{\partial x} \sin \theta \sin \varphi \quad \text { at } \quad y=0 \quad \text { and } y=A_{y}, \\
\frac{\partial W_{x}}{\partial z} & =-\frac{\partial T}{\partial x} \cos \theta \quad \text { at } z=0 \quad \text { and } z=1, \\
\frac{\partial W_{y}}{\partial x} & =\frac{\partial T}{\partial x} \sin \theta \sin \varphi \quad \text { at } \quad x=0 \quad \text { and } x=A_{x}, \\
\frac{W_{y}}{\partial W_{y}} & =0 \quad \text { at } \quad y=0 \quad \text { and } \quad y=A_{y}, \\
\frac{\partial W_{z}}{\partial x} & =\frac{\partial T}{\partial y} \cos \theta \quad \text { at } \quad z=0 \quad \text { and } \quad z=1, \\
\frac{\partial W_{z}}{\partial y} & =-\frac{\partial T}{\partial z} \sin \theta \sin \varphi \quad \text { at } \quad x=0 \quad \text { and } \quad x=A_{x}, \\
W_{z} & =0 \quad \text { at } \quad z=0 \quad \text { and } z=1 .
\end{aligned}
$$

Finally, for the temperature, we have

$$
\begin{aligned}
T & =-A_{x} / 2 \quad \text { at } \quad x=0 \quad \text { and } T=A_{x} / 2 \quad \text { at } x=A_{x}, \\
\frac{\partial T}{\partial y} & =0 \quad \text { at } \quad y=0 \quad \text { and } \quad y=A_{y}, \\
\frac{\partial T}{\partial z} & =0 \quad \text { at } \quad z=0 \quad \text { and } z=1 .
\end{aligned}
$$

For simplicity, when the vibration vector $\boldsymbol{n}$ is contained in one of the principal planes of the cavity $x z, x y$, and $y z$, the corresponding vibration is denoted as $x z$ vibration, $x y$ vibration, and $y z$ vibration, and the vibration angles, which are thus defined in these planes with respect to the first axis of these planes, are $\alpha_{x z}, \alpha_{x y}$, and $\alpha_{y z}$, respectively. For example, $\alpha_{x z}$ is the angle between the $x$ axis and the unit vector $\boldsymbol{n}$ for vibrations with a direction contained in the $x z$ plane. Note that the vibration angles $\alpha$ are related to the angles $\varphi$ and $\theta$ by the following relations:

$\varphi=0$ and $\theta+\alpha_{x z}=\pi / 2$ for $x z$ vibration,

$\varphi=\alpha_{x y}$ and $\theta=\pi / 2$ for $x y$ vibration,

$\varphi=\pi / 2$ and $\theta+\alpha_{y z}=\pi / 2$ for $y z$ vibration.

Equations (7)-(12) can then be written as a function of either $\alpha_{x y}, \alpha_{x z}$, or $\alpha_{y z}$ only in each of these three cases.

Some specific directions of vibration are common to two planes (intersection between these two planes) and they correspond to the principal axes, i.e., the $x$ axis, $y$ axis, and $z$ axis. More precisely, vibration in the $x$ direction can be found for $\alpha_{x y}=0$ and $\alpha_{x z}=0$, vibration in the $y$ direction can be 


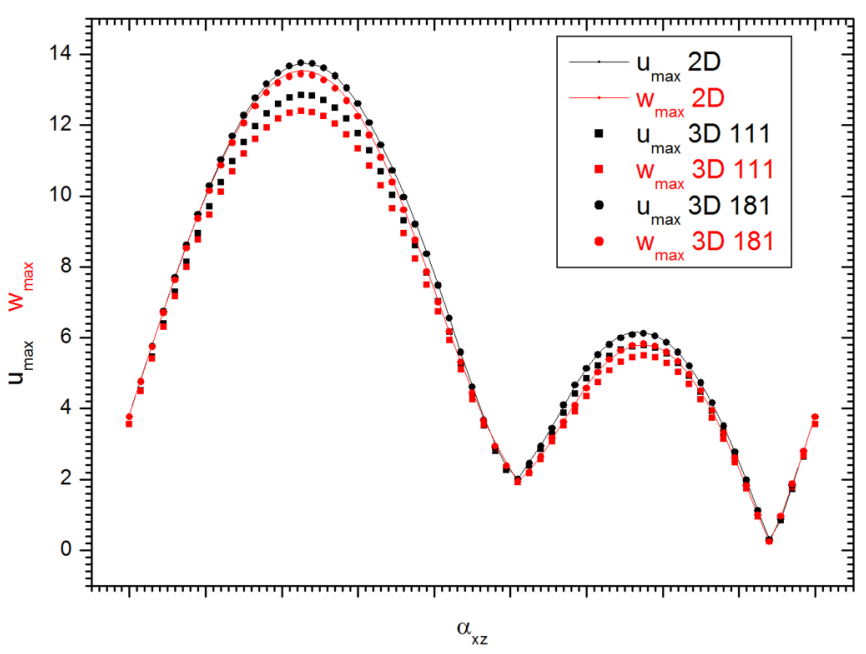

FIG. 2. Comparison of the maximum horizontal (along $x$ ) and vertical (along $z$ ) velocity components, $u_{\max }$ and $w_{\max }$, obtained by $2 \mathrm{D}$ and $3 \mathrm{D}$ computations for $x z$ vibrations in all possible directions $(\mathrm{Gr}=1000$ and $\left.\mathrm{Gr}_{\mathrm{v}}=10000\right)$. The 3D computations are performed for a $1 \times 1 \times 1$ cubic cell and a $1 \times 8 \times 1$ rectangular duct. Due to symmetry properties, the curves of minimum velocity have exactly the same variations, but with an opposite sign.

found for $\alpha_{x y}=\pi / 2$ and $\alpha_{y z}=0$, and vibration in the $z$ direction can be found for $\alpha_{x z}=\pi / 2$ and $\alpha_{y z}=\pi / 2$.

The accurate spectral collocation method [24] has been used to solve numerically the system of Eqs. (3)-(5) and (7)-(12). Such a method already showed its efficiency in the investigation of the influence of high frequency vibration direction on the structure of a two-dimensional differentially heated cavity buoyant flow (Bouarab et al. [18]). The Navier-Stokes equations are solved in a primitive variables $(\boldsymbol{V}, P)$ formulation, replacing the incompressibility equation (4) by an explicit pressure Poisson equation with relevant Neumann boundary conditions derived from the NavierStokes equations, as detailed in Johnston and Liu [25] and Johnston et al. [26].

\section{VALIDATION OF THE 3D RESULTS}

Comparison of the fully three-dimensional results with those available in the literature and obtained either in 2D [18] or in 3D [21] studies shows a very good agreement. A series of computations has been performed in the case of $x z$ vibration, i.e., when the vibration vector is contained in the main flow plane ( $x z$ plane), which is also the computation domain for the two-dimensional models. The vibrations can be oriented in all possible directions, i.e., from $\alpha_{x z}=0^{\circ}$ to $\alpha_{x z}=180^{\circ}$. The thermovibrational convection results in a $1 \times 1 \times 1$ cubic cell and in a $1 \times 8 \times 1\left(A_{x}=1, A_{y}=8\right)$ cavity are compared with those obtained in a $2 \mathrm{D}$ rectangular cavity having the same aspect ratio $A_{x}=1$ (square cavity). The 2D and 3D maximum of the horizontal and vertical velocity components in the main flow plane (i.e., the $x z$ plane), namely $u_{\max }$ and $w_{\max }$, are plotted as a function of $\alpha_{x z}$ in Fig. 2 for $\mathrm{Gr}=1000$ and $\mathrm{Gr}_{\mathrm{v}}=10000$. Due to symmetry properties, these plots also correspond to the absolute values of the minimum velocities, i.e., $\left|u_{\min }\right|$ and $\left|w_{\min }\right|$.

For the $1 \times 1 \times 1$ cubic cavity, the plots of $u_{\max }$ and $w_{\max }$ with respect to $\alpha_{x z}$ are similar to those obtained in the 2D square cavity. The values of $u_{\max }$ and $w_{\max }$ obtained with the $3 \mathrm{D}$ model are found to be relatively close to those obtained with the 2D model, except in the neighborhood of the optimal vibration direction $\left(\alpha_{x z}=45^{\circ}\right)$ where the convection level reaches its maximum. This can be due to the fact that for this intense flow, the viscous effects due to the no-slip condition imposed on the walls at $y=0$ and $y=A_{y}=1$ (i.e., very close to the main flow plane at $y=A_{y} / 2=0.5$ ) are 


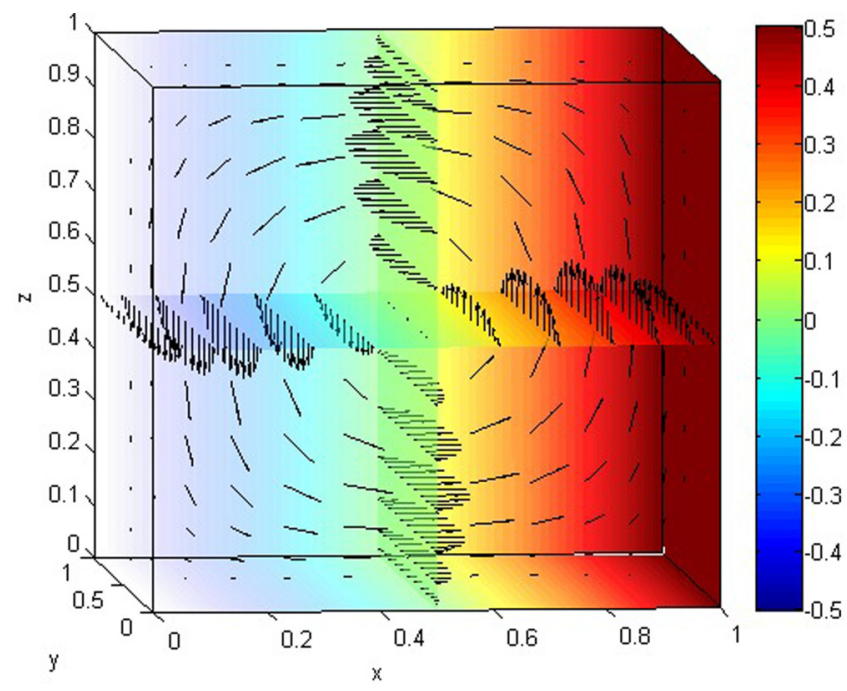

FIG. 3. Temperature and velocity fields in the reference buoyant case (without vibration) in the differentially heated cubic cell $\left(1 \times 1 \times 1\right.$ cavity) for $\mathrm{Gr}=1000\left(\mathrm{Gr}_{\mathrm{v}}=0\right)$. Left and right boundaries at $x=0$ and $x=1$ are respectively at cold and hot temperatures. Isotherms and velocity vectors are plotted at the three middle planes, $x=0.5, y=0.5$, and $z=0.5$.

significant. A better agreement is, however, found on the overall $\alpha_{x z}$ range for the $1 \times 8 \times 1$ cavity, i.e., when the transverse aspect ratio $A_{y}$ is larger and consequently the influence of viscous effects due to the lateral walls at $y=0$ and $y=A_{y}=8$ is less significant. To quantify this agreement, we have calculated the relative differences on $u_{\max }$ and $w_{\max }$ between the $3 \mathrm{D}$ results obtained for $A_{y}=8$ (i.e., in the $1 \times 8 \times 1$ cavity) and the corresponding $2 \mathrm{D}$ results. These differences are really small and remain below $1 \%$ for all the considered vibration directions.

In the following, we focus our numerical study on the case of a cubic cavity. We first describe the reference buoyant case (Sec. IV) and then present the results obtained when vibration is applied to the cavity (Sec. V).

\section{REFERENCE BUOYANT CASE}

In the reference case without vibration $\left(\mathrm{Gr}_{\mathrm{v}}=0\right)$, a buoyant flow appears in the differentially heated cubic cavity, which results from the induced horizontal temperature gradient inside the fluid. For relatively small values of the Prandtl and Grashof numbers (as $\mathrm{Pr}=0.01$ and $\mathrm{Gr}=1000$ ), the flow consists of a single convective cell. Fluid rises near the hot wall (globally in the $z$ direction), travels along the upper horizontal wall (in the $x$ direction), goes down at the cold wall (along $z$ ), and returns to the hot wall along the lower horizontal wall (along $x$ ). The resulting flow is usually called the "Hadley flow." It corresponds here to a counterclockwise convective cell which occupies the whole cavity. Temperature and flow fields corresponding to the reference case without vibration for the cubic cavity are shown in Fig. 3 for $\mathrm{Gr}=1000$ and $\mathrm{Pr}=0.01$. We see that the flow corresponds to an almost circular cell and that the temperature field remains almost diffusive. The values of $u_{\max }$ and $w_{\max }$ are very close and equal to about 3.62. This thermal convective flow has different symmetries connected with the symmetries of the geometry, the boundary conditions, and the equations themselves. Three different symmetries can be defined:

(i) a reflection symmetry $S_{P}$ (left-right symmetry) with respect to the middle vertical plane $\left(y=A_{y} / 2\right)$,

$$
S_{P}:\left(x, A_{y} / 2+y, z\right) \rightarrow\left(x, A_{y} / 2-y, z\right) \Rightarrow(u, v, w, T) \rightarrow(u,-v, w, T) ;
$$


(ii) a $\pi$-rotational symmetry $S_{A}$ with respect to the central axis parallel to the $y$ axis $\left(x=A_{x} / 2\right.$, $z=0.5)$

$$
S_{A}:\left(A_{x} / 2+x, y, 0.5+z\right) \rightarrow\left(A_{x} / 2-x, y, 0.5-z\right) \Rightarrow(u, v, w, T) \rightarrow(-u, v,-w,-T) ;
$$

(iii) a symmetry $S_{C}$ with respect to the center of the cavity $C\left(A_{x} / 2, A_{y} / 2,0.5\right)$,

$$
\begin{aligned}
S_{C}:\left(A_{x} / 2+x, A_{y} / 2+y, 0.5+z\right) & \rightarrow\left(A_{x} / 2-x, A_{y} / 2-y, 0.5-z\right) \Rightarrow(u, v, w, T) \\
& \rightarrow(-u,-v,-w,-T) .
\end{aligned}
$$

Note that the central symmetry $S_{C}$ can also be defined as the product of the two other symmetries $S_{P}$ and $S_{A}$. All these symmetries, however, can be broken when the value of $\mathrm{Gr}$ is increased. Details on the evolution of such symmetries in a $4 \times 2 \times 1$ cavity for different convection levels (increase of Gr) and different Prandtl numbers can be found in [27]. As will be explained thereafter, these symmetries can also be kept or lost depending on the direction of the applied vibration.

\section{COMPARISON OF THE EFFECT OF $x z$ VIBRATION, $x y$ VIBRATION, AND $y z$ VIBRATION}

In this section, the main features of the thermovibrational convection induced for each kind of vibration are highlighted. We will consider the buoyant flow induced in the melt contained in a $1 \times 1 \times 1$ cubic crucible for $\mathrm{Gr}=1000$ and $\mathrm{Pr}=0.01$ and investigate the effect of vibration on such flow. For each $x z$ vibration, $x y$ vibration, and $y z$ vibration, the vibrational Grashof number will be fixed to $\mathrm{Gr}_{\mathrm{v}}=10000$ and the vibration angle $\alpha$ will be varied in the whole possible range $\left[0^{\circ}, 180^{\circ}\right]$. In order to better understand this thermovibrational convection, simulations of the flows induced by vibrations in weightlessness conditions (i.e., without buoyancy force) will be first performed. The particular choice of $\mathrm{Gr}_{\mathrm{v}}$ and $\mathrm{Gr}$ with $\mathrm{Gr}_{\mathrm{v}} / \mathrm{Gr}=10$ follows our previous observations in [18] that this ratio has to be sufficiently high to induce a rich flow dynamics with, in particular, a possible flow inversion when the vibration orientation is changed. Moreover, considering a silicon melt $\left(\beta=1.1 \times 10^{-4} \mathrm{~K}^{-1}, v=3.2 \times 10^{-7} \mathrm{~m}^{2} / \mathrm{s}\right)$ submitted to a temperature gradient $\Delta T / L=10 \mathrm{~K} / \mathrm{cm}$ in a cavity of a few centimeters (for example $L=H=2.6 \mathrm{~cm}$ ), the selected value of the vibrational Grashof number $\mathrm{Gr}_{\mathrm{v}}=10000$ will correspond to vibration characteristics similar to those indicated for the experiment in [23], i.e., amplitudes $b$ in the range of $\mathrm{cm}$ down to $\mathrm{mm}$ associated with frequencies $f$ of ten to a few hundred $\mathrm{Hz}(b f \sim 0.1 \mathrm{~m} / \mathrm{s})$.

All these numerical simulations will be performed with the general system of equations (3)-(5) and (7)-(12). In particular, as indicated in (2), the general expression of the vibrational force is that given by $\mathbf{F}_{\boldsymbol{V}}=\mathrm{Gr}_{\mathrm{v}}(\boldsymbol{W} \cdot \boldsymbol{n}) \nabla T$. Note, however, that in our differentially heated cell, the temperature gradient is mainly horizontal, close to diffusive for not too strong velocities, particularly for lowPrandtl number fluids as the silicon melt, i.e., with $d T / d x \approx\left[A_{x} / 2-\left(-A_{x} / 2\right)\right] / A_{x} \approx 1$ and small values of $d T / d z$ and $d T / d y$. This indicates that the vibrational force is also mainly directed along the $x$ direction and can be approximately written as $\mathbf{F}_{\boldsymbol{V}}=\mathrm{Gr}_{\mathrm{v}}(\boldsymbol{W} \cdot \boldsymbol{n}) \mathbf{e}_{\mathbf{x}}$, where $\mathbf{e}_{\mathbf{x}}$ is the unit vector in the $x$ direction. Such approximation of the force will be used in the following to deepen our physical understanding of the numerical results.

\section{A. Flows induced by vibrations in weightlessness conditions}

The characteristics of the flows induced by vibrations in weightlessness conditions are shown in Figs. 4-9. These weightlessness flows are denoted as WF in the figures. We will present successively the results obtained for the different kinds of vibration, i.e., the $x z$ vibration, $x y$ vibration, and the $y z$ vibration.

\section{1. $x z$ vibration}

This vibration plane is vertical and perpendicular to the imposed temperature boundaries (cold and hot walls). It is also the vibration plane for the 2D model [18]. As shown in Fig. 2 for the test cases, the effects due to such vibrations, especially for large width 3D cavities, are almost the same as those obtained in the $2 \mathrm{D}$ case. 


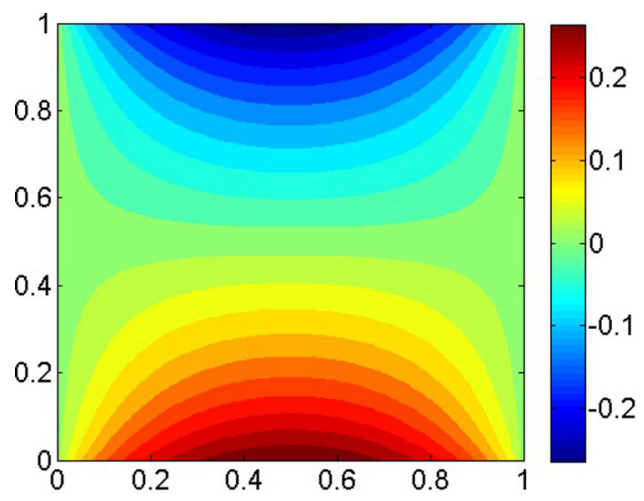

(a)

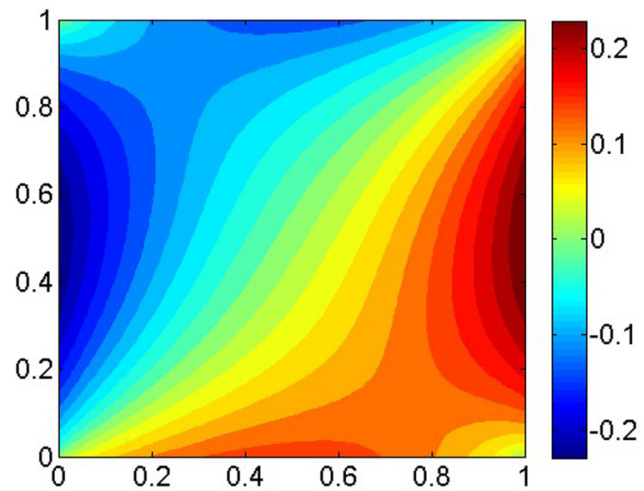

(c)

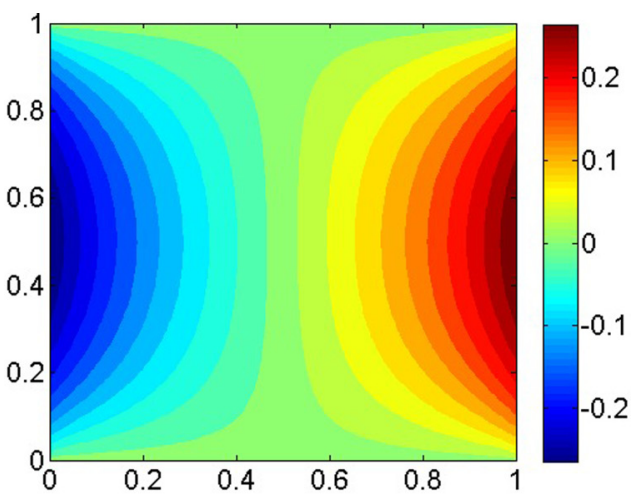

(b)

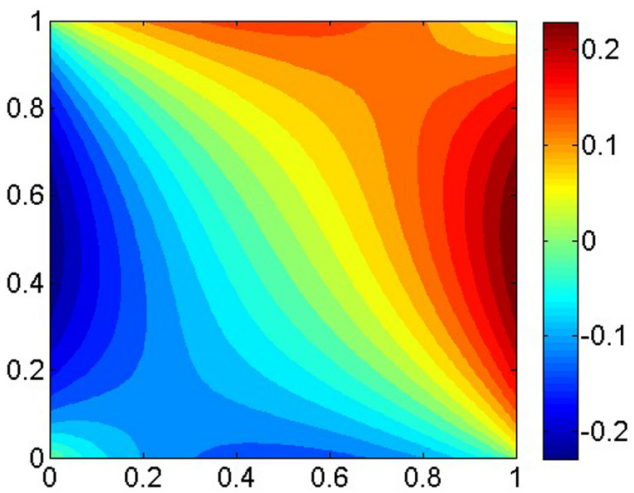

(d)

FIG. 4. Contours of pulsation velocities $W_{x}$ (a) and $W_{z}$ (b) for $\alpha_{x z}=60^{\circ}$ (identical result for $\alpha_{x z}=120^{\circ}$ ) and contours of the $x z$-vibrational force (main component of $\mathbf{F}_{V x z} / \mathrm{Gr}_{\mathrm{v}}$ along $\boldsymbol{e}_{x}$ ) for $\alpha_{x z}=60^{\circ}$ (c) and $\alpha_{x z}=$ $120^{\circ}$ (d) in the main $x z$ plane of the differentially heated cubic cell $(1 \times 1 \times 1$ cavity) for $x z$ vibration with $\mathrm{Gr}_{\mathrm{v}}=10000$ and under weightlessness conditions $(\mathrm{Gr}=0)$.

When the vibration vector $\boldsymbol{n}$ is in the $x z$ plane, the azimuthal and polar angles defining the direction of vibration are given as $\varphi=0$ and $\theta=\frac{\pi}{2}-\alpha_{x z}$ and the expression of the vibrational force $\mathbf{F}_{\boldsymbol{V}}=\mathrm{Gr}_{\mathrm{v}}(\boldsymbol{W} \cdot \boldsymbol{n}) \mathbf{e}_{\mathbf{x}}$ simplifies to

$$
\mathbf{F}_{V x z}=\mathrm{Gr}_{\mathrm{v}}\left(W_{x} \cos \alpha_{x z}+W_{z} \sin \alpha_{x z}\right) \mathbf{e}_{\mathbf{x}} .
$$

This $x z$-vibrational force involves only $W_{x}$ and $W_{z}$, i.e., the components of the pulsation velocity in the plane of vibration. Note also that, as expressed in Eq. (6), the cross product of the temperature gradient $\nabla T$ and the unit vibration vector $\boldsymbol{n}$ controls the variations of the pulsation velocity components. In the case of $x z$ vibration, it gives

$$
\begin{aligned}
& \frac{\partial W_{z}}{\partial y}-\frac{\partial W_{y}}{\partial z}=\frac{\partial T}{\partial y} \sin \alpha_{x z}, \quad \frac{\partial W_{x}}{\partial z}-\frac{\partial W_{z}}{\partial x}=\frac{\partial T}{\partial z} \cos \alpha_{x z}-\frac{\partial T}{\partial x} \sin \alpha_{x z}, \\
& \frac{\partial W_{y}}{\partial x}-\frac{\partial W_{x}}{\partial y}=-\frac{\partial T}{\partial y} \cos \alpha_{x z} .
\end{aligned}
$$

As mentioned above, $d T / d x \approx 1, d T / d z \approx 0$, and $d T / d y \approx 0$. The velocity components in the transverse direction $y$, as well as the variations of the velocity components in this $y$ direction, can 


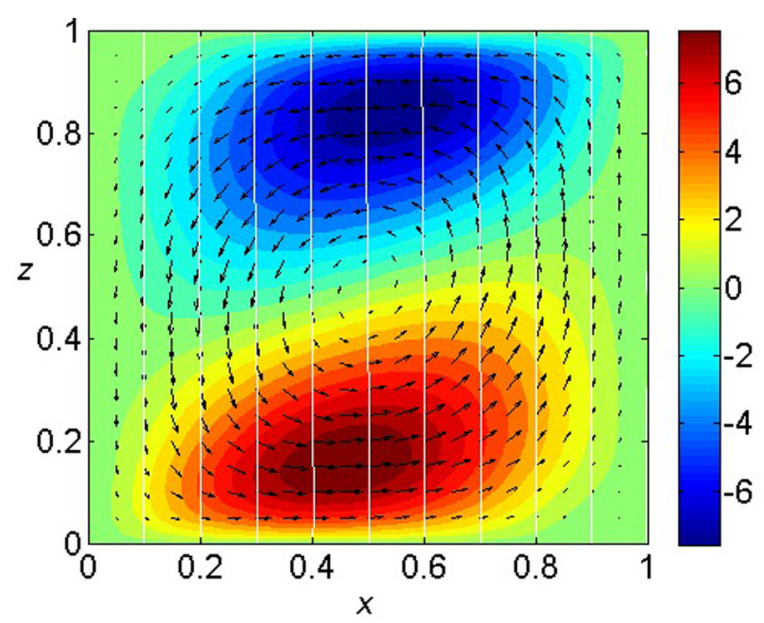

(a)

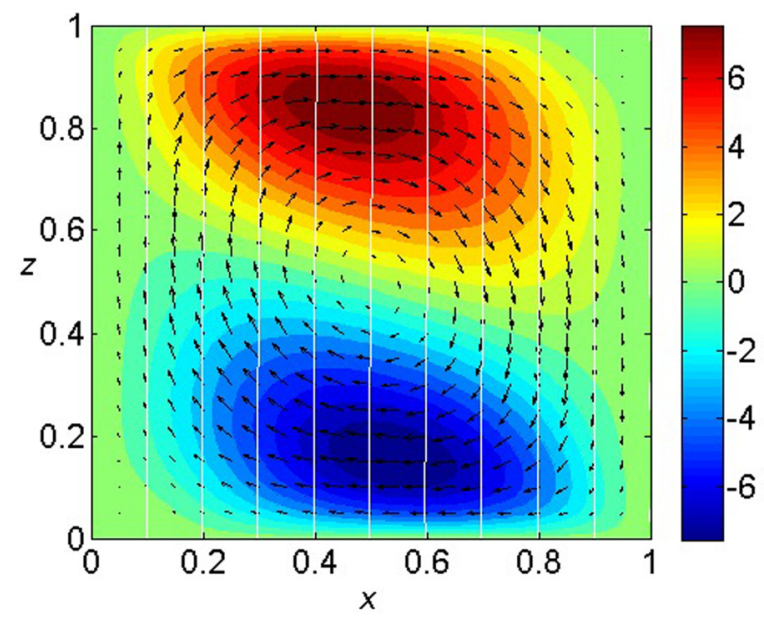

(b)

FIG. 5. Horizontal velocity contours, isotherms (white lines), and velocity vectors in the main $x z$ plane of the differentially heated cubic cell $\left(1 \times 1 \times 1\right.$ cavity) for $x z$ vibration with $\mathrm{Gr}_{\mathrm{v}}=10000$ and under weightlessness conditions $(\mathrm{Gr}=0)$ for $\alpha_{x z}=60^{\circ}$ (a) and $\alpha_{x z}=120^{\circ}$ (b).

also be considered small. The equations controlling the pulsation velocity can then be reduced to a single main equation given by

$$
\frac{\partial W_{x}}{\partial z}-\frac{\partial W_{z}}{\partial x}=-\sin \alpha_{x z}
$$

together with the continuity equation for the pulsation velocity reduced to

$$
\frac{\partial W_{x}}{\partial x}+\frac{\partial W_{z}}{\partial z}=0
$$

It is interesting to look at the spatial variation of the pulsation velocities $W_{x}$ and $W_{z}$. In Figs. 4(a) and $4(\mathrm{~b}), W_{x}$ and $W_{z}$ are plotted in the main $x z$ plane for $x z$ vibration with $\mathrm{Gr}_{\mathrm{v}}=10000$ and 


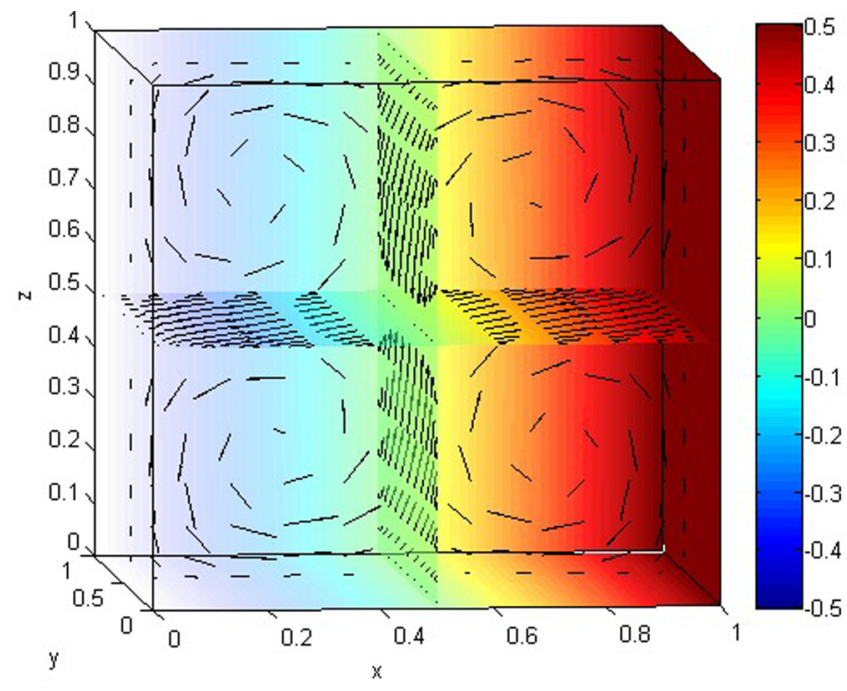

FIG. 6. Velocity and temperature fields in the differentially heated cubic cell $(1 \times 1 \times 1$ cavity $)$ under weightlessness conditions $(\mathrm{Gr}=0)$ and for $\mathrm{Gr}_{\mathrm{v}}=10000$. Vibration is applied in the $z$ direction (i.e., corresponding to $\alpha_{x z}=90^{\circ}$ or $\alpha_{y z}=90^{\circ}$ ). Left and right boundaries at $x=0$ and $x=1$ are respectively at cold and hot temperatures. Isotherms and velocity vectors are plotted at the three middle planes, $x=0.5, y=0.5$, and $z=0.5$. A four counter-rotating cells structure is obtained, the axis of the cells being parallel to the $y$ direction. Under weightlessness conditions, an exactly similar four cells structure is also obtained for vibration applied in the $y$ direction, but the cells will have their axis parallel to the $z$ direction.

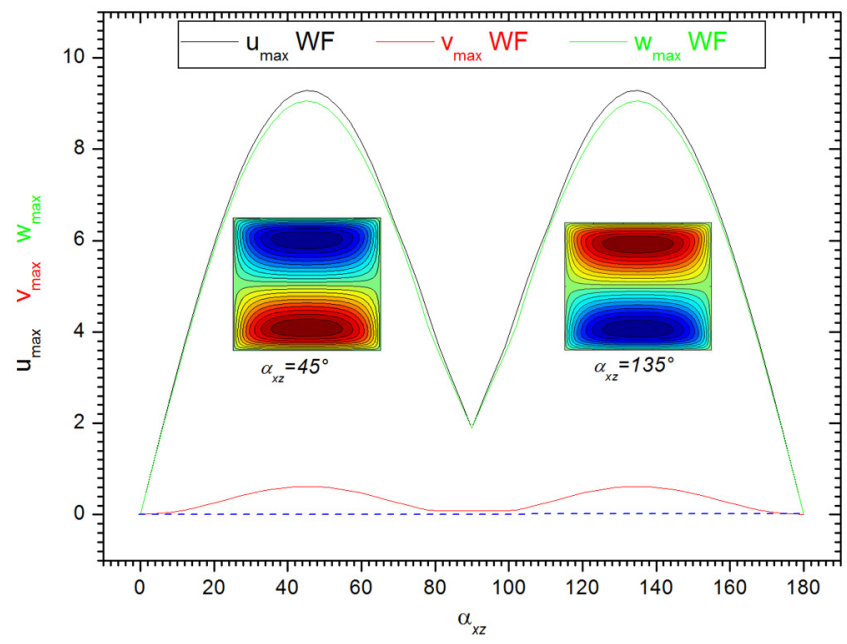

FIG. 7. Maximum velocity components in the differentially heated cubic cell $(1 \times 1 \times 1$ cavity $)$ as a function of $\alpha_{x z}$, the vibration angle in the $x z$ plane (case of $x z$ vibration) for $\mathrm{Gr}_{\mathrm{v}}=10000$ and under weightlessness conditions $\left(\mathrm{Gr}=0\right.$, flows denoted as WF for weightlessness flows). The case without vibration $\left(\mathrm{Gr}_{\mathrm{v}}=0\right)$ corresponds here to the no-flow situation (blue dashed line). Insets give contours of longitudinal velocity in the middle $y z$ plane $(x=0.5)$ for $\alpha_{x z}=45^{\circ}$ and $\alpha_{x z}=135^{\circ}$. The three curves are symmetric about $\alpha_{x z}=90^{\circ}$. This corresponds here to similar flows for $90^{\circ}-\alpha_{x z}$ and $90^{\circ}+\alpha_{x z}$, but with opposite rotation direction. These curves also give the characteristic velocities as a function of $\alpha_{x y}$ for the $x y$ vibration under weightlessness conditions. For that, the curves of $v_{\max }$ and $w_{\max }$ have to be permuted, whereas the curve of $u_{\max }$ is unchanged. 


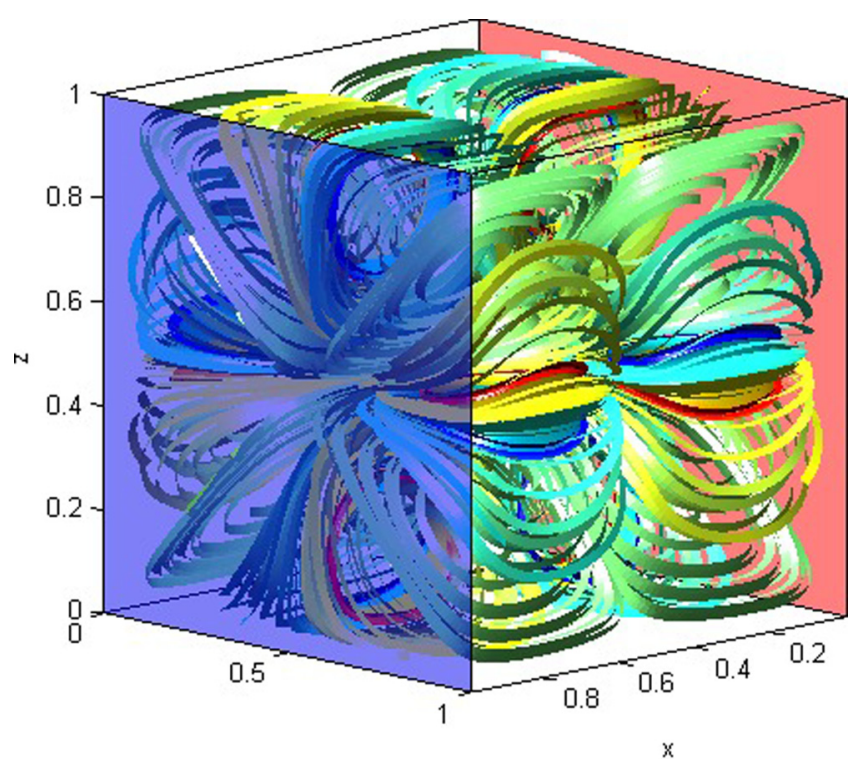

FIG. 8. 3D flow (fluid particles trajectories) in the differentially heated cubic cell $(1 \times 1 \times 1$ cavity) under weightlessness conditions $\left(\mathrm{Gr}=0\right.$ ) when $y z$ vibration is imposed in the direction $\alpha_{y z}=45^{\circ}$ with $\mathrm{Gr}_{\mathrm{v}}=10000$.

$\alpha_{x z}=60^{\circ}$ in weightlessness conditions. We see that both $\partial W_{x} / \partial z$ and $-\partial W_{z} / \partial x$ appearing in (14) have negative values and contribute together to give $-\sin \alpha_{x z}$. For the cubic cavity with $A_{x}=1$, the two pulsation velocities $W_{x}$ and $W_{z}$ are almost similar, $W_{z}$ being obtained from $W_{x}$ by a $\pi / 2$ rotation. In fact, combining (14) and (15), we get the same Laplacian equation $\partial^{2} / \partial x^{2}+\partial^{2} / \partial z^{2}=0$ for $W_{x}$

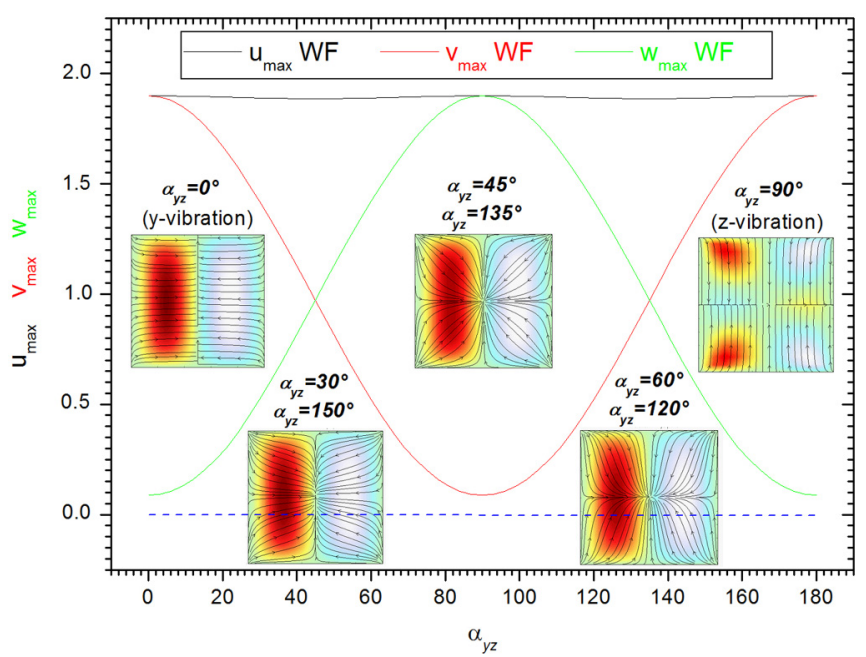

FIG. 9. Maximum velocity components in the differentially heated cubic cell $(1 \times 1 \times 1$ cavity $)$ as a function of $\alpha_{y z}$, the vibration angle in the $y z$ plane (case of $y z$ vibration) for $\mathrm{Gr}_{\mathrm{v}}=10000$ and under weightlessness conditions ( $\mathrm{Gr}=0$, flows denoted as WF for weightlessness flows). The case without vibration $\left(\mathrm{Gr}_{\mathrm{v}}=0\right)$ corresponds here to the no-flow situation (blue dashed line). Insets give contours of transverse velocity $v$ and streamlines in the middle $y z$ plane $(x=0.5)$ and for different values of $\alpha_{y z}$. The three curves are symmetric about $\alpha_{y z}=90^{\circ}$. This corresponds here to exactly similar flows for $90^{\circ}-\alpha_{y z}$ and $90^{\circ}+\alpha_{y z}$. 
and $W_{z}$, and the respective boundary conditions given in Sec. II actually lead to similar fields within a $\pi / 2$ rotation. The presence of $\sin \alpha_{x z}$ in these boundary conditions, as in the right-hand side of (14), also indicates that $W_{x}$ and $W_{z}$ remain unchanged when the value of $\alpha_{x z}$ changes from $90^{\circ}-\alpha$ to $90^{\circ}+\alpha$. To confirm this result, we checked that the plots of $W_{x}$ and $W_{z}$ obtained for $\alpha_{x z}=120^{\circ}$ are exactly the same as those corresponding to $\alpha_{x z}=60^{\circ}$ [Figs. 4(a) and 4(b)].

For the 2D model [18], it was shown that, when the vibration effects are dominant $\left(\mathrm{Gr}_{\mathrm{v}} \gg \mathrm{Gr}\right)$, the flow obtained for $\alpha_{x z}=90^{\circ}+\alpha$ is similar to that obtained for $\alpha_{x z}=90^{\circ}-\alpha$, but with opposite rotation direction, corresponding in fact to an up-down symmetry between these flows. We can reasonably assume that such a result is also valid for the 3D case with $x z$ vibration when weightlessness conditions are considered. If $T_{1}, W_{x, 1}$, and $W_{z, 1}$ are the fields defined at $x_{1}, y_{1}$, and $z_{1}$ and solutions for $\alpha_{x z}=90^{\circ}-\alpha$, the fields obtained for $\alpha_{x z}=90^{\circ}+\alpha$ and defined at $x_{2}=x_{1}, y_{2}=y_{1}$, and $z_{2}=-z_{1}$ are $T_{2}=T_{1}, W_{x, 2}=-W_{x, 1}$, and $W_{z, 2}=W_{z, 1}$. The corresponding forces $\mathbf{F}_{\boldsymbol{V} x z, 1}$ and $\mathbf{F}_{V x z, 2}$ in the general case are respectively

$$
\begin{aligned}
\mathbf{F}_{V x z, 1} & =\mathrm{Gr}_{\mathrm{v}}\left[W_{x, 1} \cos \left(90^{\circ}-\alpha\right)+W_{z, 1} \sin \left(90^{\circ}-\alpha\right)\right] \nabla T_{1} \\
& =\mathrm{Gr}_{\mathrm{v}}\left[W_{x, 1} \sin (\alpha)+W_{z, 1} \cos (\alpha)\right] \nabla T_{1}
\end{aligned}
$$

and

$$
\begin{aligned}
\mathbf{F}_{V x z, 2} & =\mathrm{Gr}_{\mathrm{v}}\left[W_{x, 2} \cos \left(90^{\circ}+\alpha\right)+W_{z, 2} \sin \left(90^{\circ}+\alpha\right)\right] \nabla T_{2} \\
& =\operatorname{Gr}_{\mathrm{v}}\left\{-W_{x, 1}[-\sin (\alpha)]+W_{z, 1} \cos (\alpha)\right\} \nabla T_{1}=\mathbf{F}_{V x z, 1} .
\end{aligned}
$$

For example, this shows that the force obtained for $\alpha_{x z}=90^{\circ}+\alpha$ is the up-down symmetric of the force obtained for $\alpha_{x z}=90^{\circ}-\alpha$. This is illustrated in Figs. 4(c) and 4(d) by the plot of the forces obtained for $\alpha_{x z}=60^{\circ}$ and $120^{\circ}$. Such forces will generate similar flows, with up-down symmetry between them and opposite rotation direction. These flows will have the same characteristics, e.g., the same maximal velocities. Such symmetric flows are illustrated in Figs. 5(a) and 5(b) by the plots of the velocity vectors in the $x z$ plane for the cases $\alpha_{x z}=60^{\circ}$ and $120^{\circ}$ in weightlessness conditions. Note that these single cell flows in the $x z$ plane have the same symmetries as the buoyant flow, i.e., $S_{p}, S_{A}$, and $S_{C}$ symmetries. This result is valid for the whole $\alpha_{x z}$ range from $0^{\circ}$ to $180^{\circ}$. When the $x z$ vibration will be applied to the buoyant flow, it is then expected that all these symmetries will still be found.

We can consider the particular cases corresponding to $\alpha_{x z}=0^{\circ}$ and $90^{\circ}$. When vibration is imposed in the $x$ direction $\left(\alpha_{x z}=0^{\circ}\right)$, Eq. (14) gives $\partial W_{x} / \partial z-\partial W_{z} / \partial x=0$ and the boundary conditions give $\partial W_{x} / \partial z=0$ and $\partial W_{z} / \partial x=0$. Both $W_{x}$ and $W_{z}$ are then expected to be very small and the vibrational force, reduced to $\mathbf{F}_{V x z}\left(0^{\circ}\right)=\mathbf{F}_{V \boldsymbol{x}} \approx \mathrm{Gr}_{\mathrm{v}} W_{x} \mathbf{e}_{\mathbf{x}}$, is also very small, leading then to a very weak flow.

When vibration is imposed in the $z$ direction $\left(\alpha_{x z}=90^{\circ}\right)$, Eq. (14) gives $\partial W_{x} / \partial z-\partial W_{z} / \partial x=$ -1 and the boundary conditions give $\partial W_{x} / \partial z=-1$ and $\partial W_{z} / \partial x=1$. The pulsation velocities are similar to those shown in Figs. 4(a) and 4(b), but they have their maximal magnitude [maximal value close to 0.3, compared to 0.25 in Figs. 4(a) and 4(b) for $\alpha_{x z}=60^{\circ}$ ]. However, only the component $W_{z}$ is included in the vibration force as $\mathbf{F}_{V x z}\left(90^{\circ}\right)=\mathbf{F}_{V z} \approx \mathrm{Gr}_{\mathrm{v}} W_{z} \mathbf{e}_{\mathbf{x}}$. This vibrational force, mainly along $x$, depends only on the vertical pulsation velocity $W_{z}$, which has opposite sign in the right and left parts (along $x$ ) of the cavity. This horizontal force, opposite in the right and left parts of the cavity and more intense at midheight, will not generate a usual single cell flow, but a symmetric four counter-rotating cells flow (Fig. 6). The cells have their axis parallel to the $y$ direction. The velocities go outwards at midheight, so that the upper-left and lower-right cells have a clockwise rotation in $x z$ planes whereas the upper-right and lower-left cells have a counterclockwise rotation. Due to the opposite forces involved, the resulting flow will also have a rather limited intensity. Such four cells flow was also observed by Farooq and Homsy [22], Savino et al. [21], and Shevtsova et al. [23]. 
The variation of the characteristic velocities $u_{\max }, v_{\max }$, and $w_{\max }$ as a function of $\alpha_{x z}$ for the $x z$ vibration in weightlessness conditions is given in Fig. 7. The three curves also correspond to $\left|u_{\min }\right|$, $\left|v_{\text {min }}\right|$, and $\left|w_{\text {min }}\right|$, respectively. We see that the curves of the characteristic velocities are symmetric with respect to $\alpha_{x z}=90^{\circ}$. In fact, as shown before and as indicated by the flows given as insets, the flow has opposite rotation direction below and above $\alpha_{x z}=90^{\circ}$. For these single cell flows, we see that the vertical velocity is slightly smaller than the horizontal velocity, especially around $\alpha_{x z}=45^{\circ}$ and $135^{\circ}$ where they have their maximal intensities. In contrast, the transverse component remains small.

\section{2. $x y$ vibration}

This $x y$-vibration plane is perpendicular to the imposed temperature boundaries, as was the $x z$ vibration plane. In the absence of buoyancy, the $x y$ vibration is then expected to give exactly the same effects as the $x z$ vibration, with only a change of plane corresponding to a $\pi / 2$ rotation around the $x$ axis. The flows that have principally been obtained in the $x z$ plane when $\alpha_{x z}$ is changed for the $x z$ vibration are now obtained in the $x y$ plane when $\alpha_{x y}$ is changed for the $x y$ vibration. The same characteristic behaviors and velocities are thus obtained, and the results presented for the $x z$ vibration in the previous subsection can all be transposed to give the results obtained for the $x y$ vibration [e.g., $w_{\max }\left(v_{\max }\right)$ as a function of $\alpha_{x z}$ for the $x z$ vibration in Fig. 7 also gives $v_{\max }\left(w_{\max }\right)$ as a function of $\alpha_{x y}$ for the $x y$ vibration, whereas the curve of $u_{\max }$ is similar].

For $x y$ vibration, the flow then consists mainly in a single cell flow, which, however, occurs in the $x y$ plane and changes its rotation direction when $\alpha_{x y}$ is below or above $90^{\circ}$. For $\alpha_{x y}=90^{\circ}$, we obtain the four counter-rotating cells flow, but the cells have now their axis parallel to the $z$ direction (see Fig. 6 and its caption). Note that the symmetries of such flow are different from those of the buoyant flow: a reflection symmetry with respect to the middle $x y$ plane, a $\pi$-rotation symmetry with respect to the middle $z$ axis, and the central symmetry. When the $x y$ vibration will be applied to the buoyant flow, it is then expected that the only symmetry that will still hold is the central symmetry.

\section{3. $y z$ vibration}

The $y z$ vibration plane is different from the previous vibration planes, as it is parallel to the cold and hot walls and is consequently at an almost uniform temperature, particularly for small Prandtl numbers.

In the case of $y z$ vibration, we have $\varphi=\pi / 2$ and $\theta+\alpha_{y z}=\pi / 2$, and the vibrational force $\mathbf{F}_{V \boldsymbol{y z}}$ involves then vertical and transverse pulsation velocities $W_{z}$ and $W_{y}$ :

$$
\mathbf{F}_{V y z}=\mathrm{Gr}_{\mathrm{v}}\left(W_{y} \cos \alpha_{y z}+W_{z} \sin \alpha_{y z}\right) \nabla T \text {. }
$$

This force would be mainly horizontal (as for $\mathbf{F}_{V x z}$ and $\mathbf{F}_{V x y}$ ) and can be written as

$$
\mathbf{F}_{V y z}=\operatorname{Gr}_{\mathrm{v}}\left(W_{y} \cos \alpha_{y z}+W_{z} \sin \alpha_{y z}\right) \mathbf{e}_{\mathbf{x}} .
$$

It is clear that $\mathbf{F}_{V y z}$ is perpendicular to the $y z$-vibration plane and consequently it will act in a different way compared to $\mathbf{F}_{\boldsymbol{V} x z}$ and $\mathbf{F}_{\boldsymbol{V} \boldsymbol{x} y}$.

Since $\partial T / \partial y \approx 0, \partial T / \partial z \approx 0$, and $\partial T / \partial x \approx 1$, the cross product of temperature gradient $\nabla T$ and $y z$-vibration unit vector $\boldsymbol{n}$ results in the following expressions for the pulsation velocity:

$$
\begin{gathered}
\frac{\partial W_{z}}{\partial y}-\frac{\partial W_{y}}{\partial z}=\frac{\partial T}{\partial y} \sin \alpha_{y z}-\frac{\partial T}{\partial z} \cos \alpha_{y z} \approx 0, \\
\frac{\partial W_{x}}{\partial z}-\frac{\partial W_{z}}{\partial x}=-\sin \alpha_{y z}, \\
\frac{\partial W_{y}}{\partial x}-\frac{\partial W_{x}}{\partial y}=\cos \alpha_{y z} .
\end{gathered}
$$

Differently from the $x z$ and $x y$ vibrations, Eq. (17) relating the pulsation velocity components in the plane of vibration $\left(W_{y}\right.$ and $\left.W_{z}\right)$ is independent of the temperature gradient along $x$ for $y z$ vibration. 
Their derivatives in the plane of vibration are expected to be small and their main variations are expected to be along $x$, as expressed in (18) and (19).

The two particular cases corresponding to $\alpha_{y z}=90^{\circ}$ ( $z$ vibration) and $0^{\circ}$ ( $y$ vibration) have already been considered in the case of $x z$ and $x y$ vibration, respectively. For $\alpha_{y z}=90^{\circ}$, a four cells flow with axis parallel to $y$ has been obtained in connection with the vibrational force which, as $W_{z}$, varies along $x$ ( $W_{y}$ is very weak in this case), and similarly for $\alpha_{y z}=0^{\circ}$, a four cells flow with axis parallel to $z$ is obtained in connection with the vibrational force which, as $W_{y}$, varies along $x$ ( $W_{z}$ is very weak in this case). For angles $\alpha_{y z}$ between these two values, the vibrational force will depend on both $W_{z}$ and $W_{y}$, which also vary in intensity with $\alpha_{y z}$, and the four cells will have intermediate orientations connecting the two specific orientations at $\alpha_{y z}=90^{\circ}$ and $\alpha_{y z}=0^{\circ}$. Such behavior is illustrated in Fig. 8 for $\alpha_{y z}=45^{\circ}$ : in this 3D view of streamlines, we clearly see the intermediate orientation of the cells, here approximately along the diagonal of the $y z$ plane. The progressive change of the cells' orientation can also be observed in the insets of Fig. 9 where the streamlines are plotted in the middle $y z$ plane.

For $\alpha_{y z}=180^{\circ}$ the flow consists of the same four cells flow as for $\alpha_{y z}=0^{\circ}$. Consequently, we can reasonably expect that the transition from $\alpha_{y z}=90^{\circ}$ to $180^{\circ}$ will be the same as that from $\alpha_{y z}=90^{\circ}$ to $0^{\circ}$. To go further, we will compare the situations at $\alpha_{y z}=90^{\circ}-\alpha$ and $90^{\circ}+\alpha$. When $\alpha_{y z}$ is changed from $90^{\circ}-\alpha$ to $90^{\circ}+\alpha, W_{z}$ is not changed and $W_{y}$ is changed to its opposite in agreement with the properties of the sine and cosine functions in (18) and (19), respectively. The vibrational forces for $90^{\circ}-\alpha$ and $90^{\circ}+\alpha$ are found to be equal. Indeed, we have

$$
\begin{aligned}
\mathbf{F}_{\boldsymbol{V} \boldsymbol{y z}}\left(90^{\circ}+\alpha\right) & =\mathrm{Gr}_{\mathrm{v}}\left[W_{y}\left(90^{\circ}+\alpha\right) \cos \left(90^{\circ}+\alpha\right)+W_{z}\left(90^{\circ}+\alpha\right) \sin \left(90^{\circ}+\alpha\right)\right] \nabla T \\
& =\mathrm{Gr}_{\mathrm{v}}\left\{-W_{y}\left(90^{\circ}-\alpha\right)[-\sin (\alpha)]+W_{z}\left(90^{\circ}-\alpha\right) \cos (\alpha)\right\} \nabla T \\
& =\mathrm{Gr}_{\mathrm{v}}\left[W_{y}\left(90^{\circ}-\alpha\right) \cos \left(90^{\circ}-\alpha\right)+W_{z}\left(90^{\circ}-\alpha\right) \sin \left(90^{\circ}-\alpha\right)\right] \nabla T \\
& =\mathbf{F}_{\boldsymbol{V} \boldsymbol{y z}}\left(90^{\circ}-\alpha\right) .
\end{aligned}
$$

This indicates that, for two orientations $\alpha_{y z}$ which are symmetric with respect to $90^{\circ}$, the same vibrational force will be obtained, which will consequently generate the same flow. The equality of the flow intensity obtained for the vibration orientations at $\alpha_{y z}=90^{\circ}-\alpha$ and $90^{\circ}+\alpha$ is clearly highlighted by the symmetry of the curves of $u_{\max }, v_{\max }$, and $w_{\max }$ about the value $\alpha_{y z}=90^{\circ}$ in Fig. 9. We have also verified that the corresponding flow patterns are identical (see the insets in Fig. 9 allowing the comparison for $\alpha_{y z}=30^{\circ}$ and $150^{\circ}$, for $\alpha_{y z}=45^{\circ}$ and $135^{\circ}$, and for $\alpha_{y z}=60^{\circ}$ and $120^{\circ}$ ). Note that $u_{\max }$ is almost constant in the whole $\alpha_{y z}$ range, corresponding to the fact that the horizontal motion is always present for the different roll orientations. The position of $u_{\max }$ in the cavity, however, must change with $\alpha_{y z}$. In contrast, $v_{\max }$ is maximal and $w_{\max }$ minimal when the rolls have a vertical axis for $\alpha_{y z}=0^{\circ}$, whereas $w_{\max }$ is maximal and $v_{\max }$ minimal when the rolls have a horizontal transverse axis for $\alpha_{y z}=90^{\circ}$.

The symmetries of the flows obtained with $y z$ vibration are numerous: reflection symmetries with respect to the three middle planes, $\pi$-rotation symmetries with respect to the three middle axes, and the central symmetry. As these symmetries include those of the buoyant flow, it is expected that, when the $y z$ vibration will be applied to the buoyant flow, the typical symmetries of the latter will be retained.

\section{B. Buoyant flows submitted to vibrations}

The main results obtained for buoyant flows submitted to vibrations are shown in Figs. 10-12 $\left(\mathrm{Gr}=1000\right.$ and $\left.\mathrm{Gr}_{\mathrm{v}}=10000\right)$. In order to be able to compare the results between the different vibration orientations, we have plotted the variation of the characteristic velocities for the different vibration orientations on a same figure, namely in Fig. 10 for $u_{\max }$, in Fig. 11 for $w_{\max }$ and in Fig. 12 for $v_{\max }$. These variations are given as solid lines, whereas the results in weightlessness conditions are recalled as dashed lines. In addition, we have illustrated the flows corresponding to the $x y$ vibration in Fig. 10, the $x z$ vibration in Fig. 11, and the $y z$ vibration in Fig. 12 by insets. 


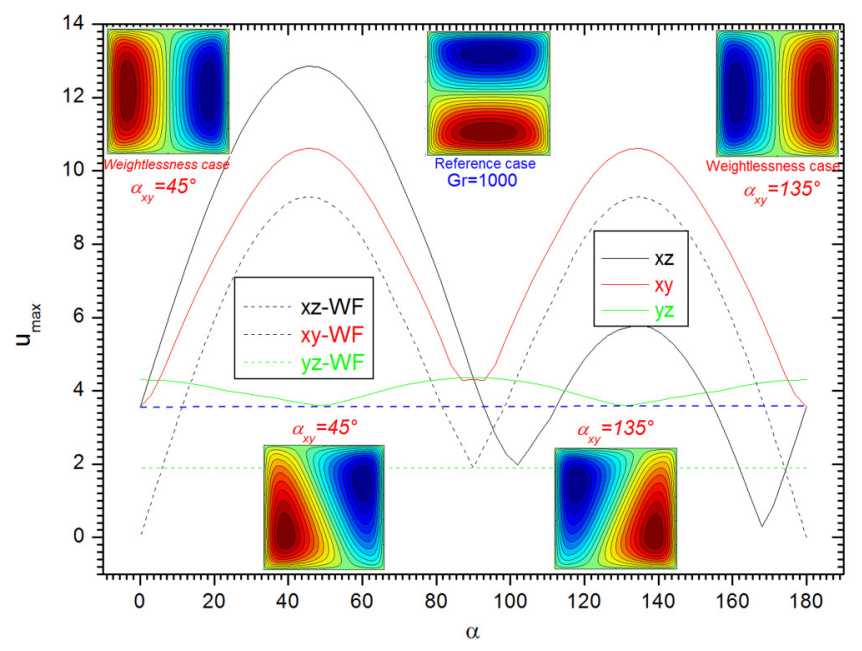

FIG. 10. Maximum longitudinal velocity $u_{\max }$ (along $\left.x\right)$ in the differentially heated cubic cell $(1 \times 1 \times 1$ cavity) as a function of the vibration angle $\alpha$, when the vibration vector is contained in one of the three principal planes ( $x z, x y$, and $y z$ planes) for $\mathrm{Gr}=1000$ and $\mathrm{Gr}_{\mathrm{v}}=10000$. The results are given with solid lines of different colors. The corresponding results in weightlessness conditions (denoted as WF) are also given as dashed lines for comparison (note that the same curve, plotted as black dashed line, is obtained for $x z$ and $x y$ vibration). Blue dashed line corresponds to the pure buoyant flow (reference case with $\mathrm{Gr}_{\mathrm{v}}=0$ ). Insets give contours of the longitudinal velocity $u$ in the middle $y z$ plane $(x=0.5)$ for the reference buoyant case $\left(\mathrm{Gr}_{\mathrm{v}}=0\right)$ and for the pure vibrational cases (weightlessness cases, $\left.\mathrm{Gr}=0\right)$ and convectovibrational cases for $x y$ vibration with angles $\alpha_{x y}=45^{\circ}$ and $135^{\circ}$.

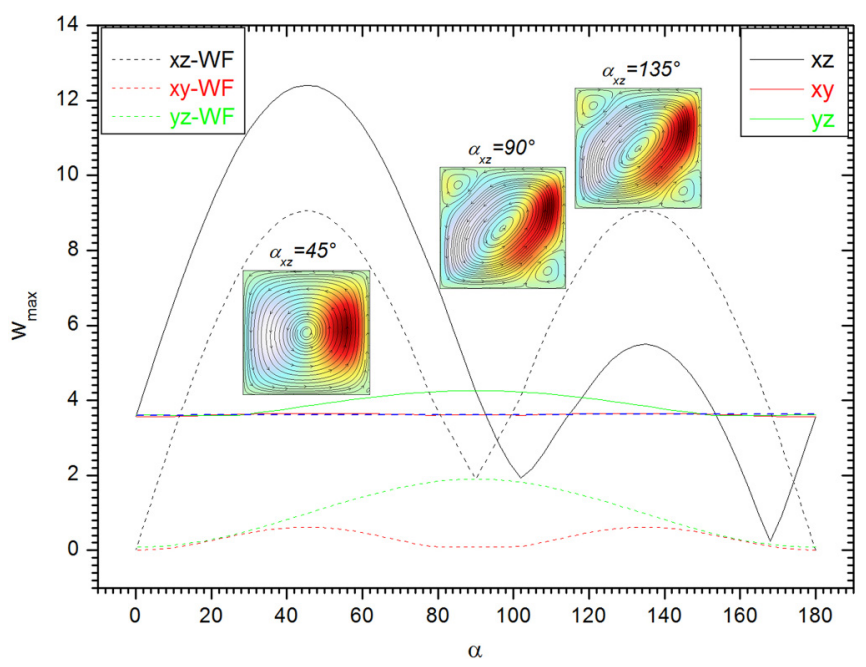

FIG. 11. Maximum vertical velocity $w_{\max }$ (along $\left.z\right)$ in the differentially heated cubic cell $(1 \times 1 \times 1$ cavity) as a function of the vibration angle $\alpha$, when the vibration vector is contained in one of the three principal planes ( $x z, x y$, and $y z$ planes) for $\mathrm{Gr}=1000$ and $\mathrm{Gr}_{\mathrm{v}}=10000$. The results are given with solid lines of different colors. The corresponding results in weightlessness conditions (denoted as WF) are also given as dashed lines for comparison. Blue dashed line corresponds to the pure buoyant flow (reference case with $\mathrm{Gr}_{\mathrm{v}}=0$ ). Insets give contours of the vertical velocity $w$ and streamlines in the middle $x z$ plane $(y=0.5)$ for $x z$ vibration with angles $\alpha_{x z}=45^{\circ}, 90^{\circ}$, and $135^{\circ}$. 


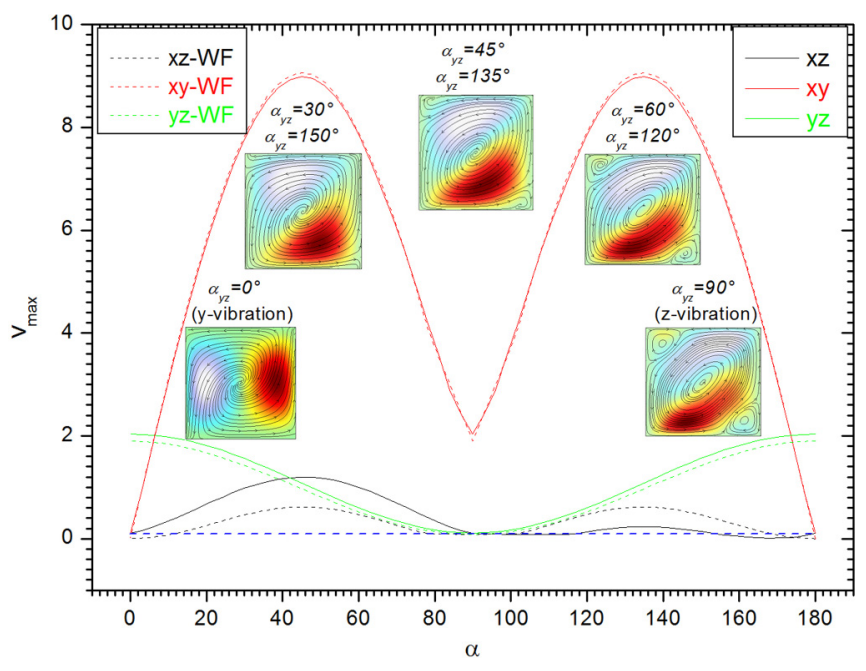

FIG. 12. Maximum transverse velocity $v_{\max }$ (along $\left.y\right)$ in the differentially heated cubic cell $(1 \times 1 \times 1$ cavity) as a function of the vibration angle $\alpha$, when the vibration vector is contained in one of the three principal planes ( $x z, x y$, and $y z$ planes) for $\mathrm{Gr}=1000$ and $\mathrm{Gr}_{\mathrm{v}}=10000$. The results are given with solid lines of different colors. The corresponding results in weightlessness conditions (denoted as WF) are also given as dashed lines for comparison. Blue dashed line corresponds to the pure buoyant flow (reference case with $\mathrm{Gr}_{\mathrm{v}}=0$ ). Insets give contours of the longitudinal velocity $u$ and streamlines in the middle $x z$ plane $(y=0.5)$ for $y z$ vibration with different angles $\alpha_{y z}$.

\section{1. $x z$ vibration}

For buoyant flows submitted to $x z$ vibration, the variations of the maximal velocities with $\alpha_{x z}$ are given as black curves in Figs. 10-12 and plots of the flow are shown as insets in Fig. 11.

For $\alpha_{x z}=0^{\circ}$ (vibration imposed in the $x$ direction), the flow is similar to the reference buoyant flow $\left(u_{\max }=w_{\max }=3.62\right)$. This result was expected since, for such vibrations, we have obtained a very weak flow in weightlessness conditions. For $\alpha_{x z}$ between $0^{\circ}$ and $90^{\circ}$, the pure thermovibrational flow, which consists in a counterclockwise cell, will enhance the buoyant flow which rotates in the same direction (inset in Fig. 11). The maximum velocity will be obtained for a value of $\alpha_{x z}$ close to $45^{\circ}$. This result is in agreement with the numerical and theoretical results obtained in the two-dimensional studies $[18,19]$. The velocity amplification achieved by vibrations is important: for example, for $\alpha_{x z}=45^{\circ}$, it leads to $u_{\max }=12.85$ (255\% increase, Fig. 10) and to $w_{\max }=12.40$ ( $243 \%$ increase, Fig. 11). Around $\alpha_{x z}=90^{\circ}$, the four cells flow obtained in weightlessness conditions will modify the circular buoyant counterclockwise cell to a diagonal counterclockwise roll, with small additional clockwise vortices near the upper left and lower right corners (Fig. 13 and inset in Fig. 11). This diagonal roll is slightly more intense than the pure buoyant flow $\left(u_{\max }=4.36\right.$ and $w_{\max }=4.26$ ). For larger values of $\alpha_{x z}$ up to $180^{\circ}$, the pure thermovibrational flow is a clockwise cell. Consequently, for this range of $\alpha_{x z}$, the vibrations will first decrease the intensity of the buoyant flow, will possibly lead to the occurrence of a clockwise cell (inset in Fig. 11) in a certain range of $\alpha_{x z}$ (the flow inversion phenomenon is here observed between about $102^{\circ}$ and $168^{\circ}$ ), and will finally lead back to the pure buoyant flow structure for $\alpha_{x z}=180^{\circ}$. The clockwise cell has its maximum intensity $\left(u_{\max }=5.79\right.$ and $\left.w_{\max }=5.46\right)$ at the middle of the inversion range, i.e., at the vicinity of $\alpha_{x z}=135^{\circ}$.

The phenomenon of flow inversion has already been studied for two-dimensional situations submitted to $x z$ vibration [18]. For a rather elongated cavity and small Prandtl number, it was shown theoretically in [18] that a necessary condition for flow inversion to occur is $\mathrm{Gr}_{\mathrm{v}} / \mathrm{Gr} \geqslant 2$ and that the limit angles $\alpha_{1}$ and $\alpha_{2}$ between which the inversion occurs verify the equation 


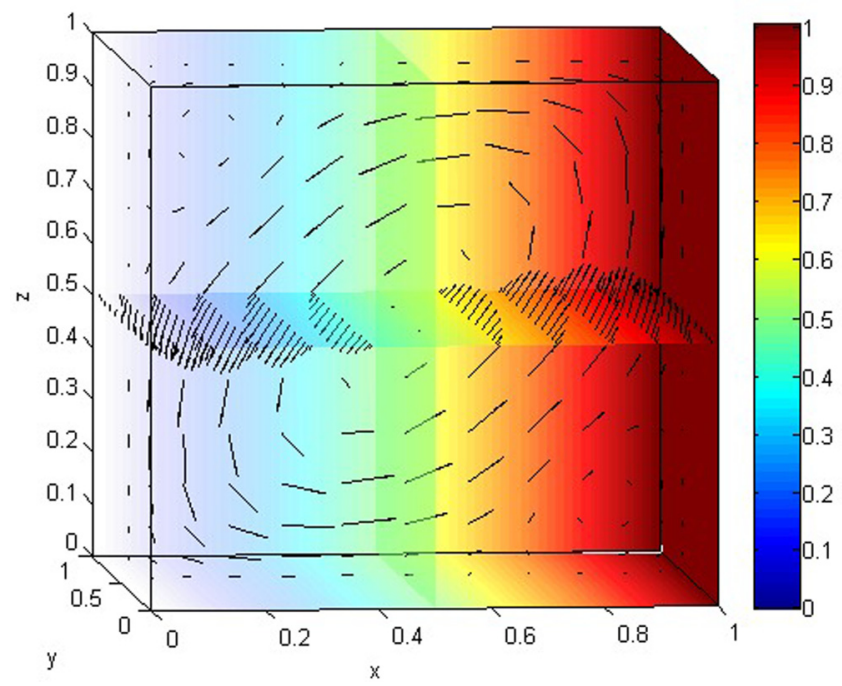

FIG. 13. Velocity and temperature fields in the differentially heated cubic cell $(1 \times 1 \times 1$ cavity $)$ for $\mathrm{Gr}=1000$ and $\mathrm{Gr}_{\mathrm{v}}=10000$ when vibration is applied in the $z$ direction (i.e., corresponding to $\alpha_{x z}=90^{\circ}$ or $\alpha_{y z}=90^{\circ}$ ). Left and right boundaries at $x=0$ and $x=1$ are respectively at cold and hot temperatures. Isotherms and velocity vectors are plotted at the three middle planes, $x=0.5, y=0.5$, and $z=0.5$.

$\mathrm{Gr}+\mathrm{Gr}_{\mathrm{v}} \sin \left(\alpha_{x z}\right) \cos \left(\alpha_{x z}\right)=0$ and are then given by

$$
\alpha_{1}=90^{\circ}+\alpha_{0} / 2 \text { and } \alpha_{2}=180^{\circ}-\alpha_{0} / 2 \quad \text { with } \alpha_{0}=\operatorname{asin}\left[2 /\left(\mathrm{Gr}_{\mathrm{v}} / \mathrm{Gr}\right)\right] .
$$

These theoretical expressions predicted very well the flow inversion obtained numerically in a cavity with $A_{x}=4$ for $\operatorname{Pr}=0.01, \mathrm{Gr}=2500$, and $\mathrm{Gr}_{\mathrm{v}}=6250$ or 62500 . We can expect that these expressions will be valid in three-dimensional buoyant flows with $x z$ vibration for elongated cavities (large $A_{x}$ ) rather than for our cubic cavity $\left(A_{x}=1\right)$. Note that, in the book of Gershuni and Lyubimov [7], a general condition is given for the quasiequilibrium states in flows affected by thermogravitational and thermovibrational effects in plane layers [see Eq. (1.114) or Eq. (1.116) in this book]. The authors consider a layer inclined at an angle $\alpha$ to the vertical (direction of the gravity) and four different orientations (vertical, longitudinal, horizontal, transversal) of the temperature gradient [expressed with the unit vector $\boldsymbol{m}\left(m_{x}, m_{z}\right)$ ] and of the vibration direction [expressed with the unit vector $\boldsymbol{n}\left(n_{x}, n_{z}\right)$ ]. For these 16 cases, they give the particular expressions of the quasiequilibrium condition. Our quasiequilibrium state corresponding to the flow inversion is not one of these 16 states as, in the situation we consider, it is the vibration direction $\alpha_{x z}$ which is continuously varied. Equation (1.116) can, however, be used to derive the quasiequilibrium condition in a plane layer corresponding to our configuration, i.e., a case where the layer is horizontal $\left(\alpha=90^{\circ}\right)$, the temperature gradient is horizontal $\left(m_{x}=0, m_{z}=1\right)$, and the vibration direction is oriented at an angle $\alpha_{x z}$ to the horizontal $\left[n_{x}=-\sin \left(\alpha_{x z}\right), n_{z}=\cos \left(\alpha_{x z}\right)\right]$. We get $\mathrm{Ra}+\mathrm{Ra}_{\mathrm{v}} \sin \left(\alpha_{x z}\right) \cos \left(\alpha_{x z}\right)=0$, i.e., the same expression we obtained in our two-dimensional rectangular cavity, only expressed with the Rayleigh number Ra rather than the Grashof number $\mathrm{Gr}\left(\mathrm{Ra}=\mathrm{Gr} \operatorname{Pr}\right.$ and $\left.\mathrm{Ra}_{\mathrm{v}}=\mathrm{Gr}_{\mathrm{v}} \operatorname{Pr}\right)$. Note also that Gershuni and Lyubimov [7] found a similar expression $\mathrm{Ra}+\mathrm{Ra}_{\mathrm{v}} \sin (\alpha) \cos (\alpha)=0$ for the quasiequilibrium condition in a plane fluid layer subjected to a horizontal temperature gradient and vertical vibrations, but the angle $\alpha$ involved in the expression is the angle of the layer to the vertical.

For the values of the Grashof and vibrational Grashof numbers considered here, $\mathrm{Gr}=1000$ and $\mathrm{Gr}_{\mathrm{v}}=10000$, the ratio $\mathrm{Gr}_{\mathrm{v}} / \mathrm{Gr}$ is 10 and thus the condition for flow inversion to occur is well satisfied. The numerical values of $\alpha_{1}$ and $\alpha_{2}$ obtained in this case and the theoretical values predicted by the model are summarized in Table I. The inversion range computed numerically is smaller than 
TABLE I. Comparison of numerical and theoretical values for flow inversion limit angles.

\begin{tabular}{ccccccc}
\hline \hline $\mathrm{Gr}$ & $\mathrm{Gr}_{\mathrm{v}}$ & $\alpha_{0} / 2$ & (theoretical) & (theoretical) & $\begin{array}{c}\alpha_{1} \\
\text { (numerical 3D) }\end{array}$ & $\begin{array}{c}\alpha_{2} \\
\text { (numerical 3D) }\end{array}$ \\
\hline 1000 & 10000 & $5.77^{\circ}$ & $95.77^{\circ}$ & $174.23^{\circ}$ & $101.81^{\circ}$ & $168.01^{\circ}$ \\
\hline \hline
\end{tabular}

the one found theoretically. This difference is due in particular to the small value of $A_{x}$ for which the hypothesis of a horizontal flow in the central part of the cavity cannot be made. Note however that, as predicted by the theoretical model, the numerical values of $\alpha_{1}$ and $\alpha_{2}$ have about the same departure from $90^{\circ}$ and $180^{\circ}$, respectively (around $11.9^{\circ}$, compared to $5.77^{\circ}$ according to the model).

In the presence of $x z$ vibration, the flow mainly remains a single cell flow with fluid circulation principally in planes parallel to the $x z$ plane and small transverse $v$ velocities. Such flow keeps all the symmetries of the buoyant flow for all $\alpha_{x z}$ values. This flow, however, can be counterclockwise as the buoyant flow, but also clockwise in a certain range of $\alpha_{x z}$. The $x z$ vibration can enhance the intensity of the buoyant flow, particularly around $\alpha_{x z}=45^{\circ}\left(u_{\max }=12.85\right)$, but can also lead to weak flows near the flow inversion limit angles. The most important decrease of the velocities is numerically observed at $\alpha_{x z}=168^{\circ}$ and is of about $93 \%\left(u_{\max }=0.28\right)$.

\section{2. xy vibration}

For buoyant flows submitted to $x y$ vibration, the variation of the maximal velocities with $\alpha_{x y}$ is shown as red curves in Figs. 10-12 and plots of the flow are shown as insets in Fig. 10. Note that, in Fig. 10, the variation of $u_{\max }$ in the weightlessness case for $x y$ vibration is the same as for the $x z$ vibration and is given as black dashed lines. Let us remind that the flow created by the $x y$ vibration in weightlessness conditions is the same as that created by the $x z$ vibration, but with a main flow plane changed from the $x z$ plane to the $x y$ plane ( $\pi / 2$ rotation). The combination of the buoyant force which creates a flow principally in the $x z$ plane and the vibrational force which creates a flow principally in the $x y$ plane is expected to lead to strongly 3D flows, with the possibility of rotating the main flow plane about the $x$ axis between the $x z$ and $x y$ orientations. The vertical velocity component of the resulting flow is of the same order of magnitude as that of the pure buoyant flow (see red solid line and blue dashed line in Fig. 11), while the transverse velocity component is of the same order of magnitude as that of the pure vibrational flow (see red solid line and red dashed line in Fig. 12).

For $\alpha_{x y}=0^{\circ}$ (vibration imposed in the $x$ direction), the flow is similar to the reference buoyant flow $\left(u_{\max }=w_{\max }=3.62\right)$. This result was expected since for such vibrations we have obtained a very weak flow in weightlessness conditions. This flow is also obtained for $\alpha_{x z}=0^{\circ}$. For $\alpha_{x y}$ between $0^{\circ}$ and $90^{\circ}$, the pure thermovibrational flow is a counterclockwise cell in the $x y$ plane with a maximum intensity reached when $\alpha_{x y}$ is close to $45^{\circ}$. In this $\alpha_{x y}$ range, the vibrational force will be responsible for the rotation of the counterclockwise cell between its initial position in the $x z$ plane (pure buoyant flow) and a position closer to the $x y$ plane (vibration dominated flow). The most important rotation is obtained for $\alpha_{x y}$ close to $45^{\circ}$ (see inset in Fig. 10). Simultaneously, the vibrations are at the origin of an increase of the longitudinal and transverse velocities (Figs. 10 and 12 , respectively) whereas the vertical velocity is almost unchanged (Fig. 11). The value of $u_{\max }$ is increased up to 10.62 , whereas $v_{\max }$ is changed from 0.11 for $\alpha_{x y}=0^{\circ}$ to 8.98 for $\alpha_{x y}=45^{\circ}$.

For $\alpha_{x y}=90^{\circ}$ (vibration imposed in the $y$ direction), the flow returns to a minimum intensity. This flow is, however, slightly different from the buoyant flow, since it is affected by the pure thermovibrational flow which corresponds to the four cells flow with axis parallel to $z$. Indeed, we observe a small enhancement of the longitudinal velocity component with $u_{\max }=4.30$ (Fig. 10) and a significant enhancement of the transverse velocity component, which is the same as for the weightlessness flow, with $v_{\max }=2.03$ (Fig. 12). With an almost unchanged vertical motion, close 


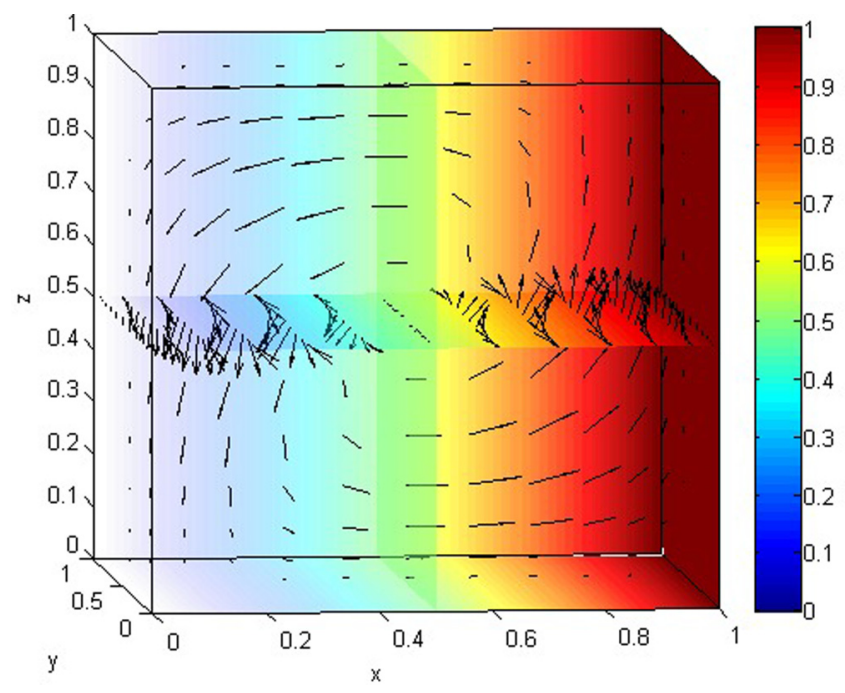

FIG. 14. Velocity and temperature fields in the differentially heated cubic cell $(1 \times 1 \times 1$ cavity $)$ for $\mathrm{Gr}=1000$ and $\mathrm{Gr}_{\mathrm{v}}=10000$ when vibration is applied in the $y$ direction (i.e., corresponding to $\alpha_{x y}=90^{\circ}$ or $\alpha_{y z}=0^{\circ}$ ). Left and right boundaries at $x=0$ and $x=1$ are respectively at cold and hot temperatures. Isotherms and velocity vectors are plotted at the three middle planes, $x=0.5, y=0.5$, and $z=0.5$.

to that induced by pure buoyancy (Fig. 11), the resulting flow structure is a perturbed $x z$ roll with increased three-dimensional effects compared to the pure buoyant flow (Fig. 14). See particularly the perturbed velocities in the middle horizontal plane.

For $\alpha_{x y}$ between $90^{\circ}$ and $180^{\circ}$, the pure thermovibrational flow is now a clockwise cell in the $x y$ plane, which, as shown previously, is the symmetric with respect to the middle $x z$ plane of what was obtained for $\alpha_{x y}$ in the first range, i.e., between $90^{\circ}$ and $0^{\circ}\left(90^{\circ}+\alpha\right.$ versus $\left.90^{\circ}-\alpha\right)$. As the pure buoyant flow has this $S_{P}$ symmetry, the resulting flow for $\alpha_{x y}$ between $90^{\circ}$ and $180^{\circ}$ will also be the symmetric with respect to the middle $x z$ plane of what was obtained for $\alpha_{x y}$ in the first range, i.e., between $90^{\circ}$ and $0^{\circ}$. For $\alpha_{x y}$ changing from $180^{\circ}$ to $90^{\circ}$, the rotation of the main flow plane will now be in the opposite direction, from its position as a counterclockwise cell in the $x z$ plane (pure buoyant flow) towards its position closer to the $x y$ plane, now as a clockwise cell in this plane (vibration dominated flow). The maximum rotation will here be obtained for $\alpha_{x y}$ close to $135^{\circ}$ (see inset in Fig. 10) and, due to the symmetry, all the characteristics of the flow can be deduced from what was obtained previously for $\alpha_{x y}$ between $0^{\circ}$ and $90^{\circ}$.

\section{3. $y z$ vibration}

For buoyant flows submitted to $y z$ vibration, the variation of the maximal velocities with $\alpha_{y z}$ is shown as green curves in Figs. 10-12 and plots of the flow are shown as insets in Fig. 12. We have previously shown that the $y z$-vibrational force $\mathbf{F}_{V y z}$ is perpendicular to the vibration plane and small compared to $\mathbf{F}_{V x z}$ and $\mathbf{F}_{V x y}$ and that the flow induced by such $y z$ vibration in weightlessness conditions is rather weak. The $y z$ vibration is thus expected to only slightly modify the buoyant flow. The results in Figs. 10 and 11 show that the main velocity components of the buoyant flow, characterized by $u_{\max }$ and $w_{\max }$, are indeed only slightly increased by the $y z$ vibration, in a really less efficient way than what can be obtained with $x z$ vibration or $x y$ vibration. However, the transverse velocity component, which was weak for the buoyant flow, can be more clearly increased (Fig. 12). The values of $v_{\max }$ are almost the same as for the weightlessness flow (compare solid and dashed green lines in Fig. 12). Note that, depending on the flow velocity components, the maximum 
amplification is induced when the $y z$ vibration is applied along the $y$ axis $\left(\alpha_{y z}=0^{\circ}\right.$ or $\left.180^{\circ}\right)$ or along the $z$ axis $\left(\alpha_{y z}=90^{\circ}\right)$.

We can have a closer look at what occurs for the different vibration angles. Remember first that the vibrational force $\mathbf{F}_{V y z}$ and the flow induced by such force in weightlessness conditions are the same for angles below and above $\alpha_{y z}=90^{\circ}, \alpha_{y z}=90^{\circ}-\alpha$, and $90^{\circ}+\alpha$, respectively. It is then only useful to discuss the results for $0^{\circ} \leqslant \alpha_{y z} \leqslant 90^{\circ}$. Moreover, the flow obtained when the $y z$ vibration is applied to the buoyant flow is expected to keep all the symmetries of the buoyant flow (see Sec. V A 3). Finally, as the changes induced by the vibration are rather weak, the global structure of the flow is expected to remain a single cell structure in the $x z$ plane, but with increased 3D effects.

As seen in Sec. V A 3, for $\alpha_{y z}=0^{\circ}$ (vibration in the $y$ direction, corresponding also to $\alpha_{x y}=90^{\circ}$ ), a four cells flow with axis $z$ is obtained in weightlessness conditions. Applied to the buoyant flow, such vibration will then affect the $u$ and $v$ velocity components. The influence on $u$, which is a main velocity of the buoyant flow, remains weak (Fig. 10). In contrast, there is a clear amplification of $v$, which is usually the weak velocity component of the buoyant flow (Fig. 12). For small values of $\alpha_{y z}$, the resulting flow structure will then be a perturbed $x z$ roll with increased three-dimensional effects compared to the pure buoyant flow (see Fig. 14). Around $\alpha_{y z}=90^{\circ}$, the four cells flow obtained in weightlessness conditions is now oriented along the $y$ axis. Such vibration will then affect the main components of the buoyant flow, the $u$ and $w$ velocity components. It will modify the circular buoyant counterclockwise cell to a diagonal counterclockwise roll, with small additional clockwise vortices near the upper left and lower right corners (Fig. 13).

As seen in Sec. V A 3, for intermediate values of $\alpha_{y z}$, the four cells of the pure vibrational case have intermediate orientations, their axis rotating between the $z$-axis and $y$-axis directions. The three velocity components of the buoyant flow will then be affected by such vibrations. When $\alpha_{y z}$ is increased from $0^{\circ}$ to $90^{\circ}$, the resulting flow will evolve with decreased three-dimensional effects and progressive evolution of the buoyant cell to a more diagonal shape and emergence of the corner vortices (see the insets in Fig. 12). Note that $u_{\max }$ becomes minimum for values of $\alpha_{y z}$ close to $45^{\circ}$ (Fig. 10). In fact, for such angles, we can think that the slight amplification of the horizontal velocity component induced by the vibrations does not occur in the zones of maximum velocity of the buoyant flow and then does not affect $u_{\max }$.

\section{DISCUSSION AND CONCLUSION}

In this paper, a three-dimensional study on the effect of high frequency vibration on a silicon melt flow (Prandtl number Pr $=0.01$ ) contained in a cubic cavity heated from the sides, under weightlessness or gravity conditions, was performed using collocation spectral method. All possible vibration directions in one of the three principal planes of the cavity were considered. The characteristic parameters are the Grashof number Gr for the buoyant flow and the vibrational Grashof number $\mathrm{Gr}_{\mathrm{v}}$ for the vibrational flow. The values chosen in this study are $\mathrm{Gr}=1000$ and $\mathrm{Gr}_{\mathrm{v}}=10000$.

It was first shown that, due to the applied horizontal temperature gradient along $x$ and the low Prandtl number, the vibrational force is principally in the $x$ direction and depends on the pulsation velocity components in the vibration plane.

In weightlessness conditions, the vibrations in a plane perpendicular to the heated and cold side walls generate a flow corresponding to a main cell in the vibration plane, this cell rotating in a certain direction for vibration angles below $90^{\circ}$ and symmetrically in the opposite direction for vibrations angles above $90^{\circ}$. Such behavior has been observed in our numerical simulations for vibrations in the $x z$ and $x y$ planes, and the results obtained are exactly similar, deduced one from the other by a $\pi / 2$ rotation around the $x$ axis. The change of the cell rotation direction at $\alpha=90^{\circ}$ goes through a four counter-rotating rolls structure with moderate intensity and with axis perpendicular to the vibration plane, i.e., $y$ axis for $x z$ vibration and $z$ axis for $x y$ vibration. These specific vibration orientations also belong to the $y z$ plane (plane parallel to the heated side walls), at $\alpha_{y z}=90^{\circ}$ and $\alpha_{y z}=0^{\circ}$, respectively. As a consequence, for $y z$ vibration between $\alpha_{y z}=0^{\circ}$ and $90^{\circ}$, the flow, which is induced by a vibrational force perpendicular to the $y z$ vibration plane, is rather weak and will 
evolve as four rolls structures with intermediate orientations of the rolls. Moreover, exactly similar results are obtained for $\alpha_{y z}$ below or above $90^{\circ}\left(90^{\circ}-\alpha\right.$ or $\left.90^{\circ}+\alpha\right)$.

In gravity conditions, the vibrations will now act on the buoyant flow. This buoyant flow corresponds to a counterclockwise cell in the $x z$ plane and has three different symmetries: a reflection symmetry with respect to the $x z$ plane, a $\pi$-rotation symmetry with respect to the central $y$ axis, and a central symmetry.

The $x z$-vibration force also generating a roll in the $x z$ plane, the resulting flow will principally be in this plane and will keep the symmetries of the buoyant flow. The resulting roll, however, will be re-enforced, in the counterclockwise direction, for vibration angles $\alpha_{x z}$ between $0^{\circ}$ and $90^{\circ}$ and will be decelerated for $\alpha_{x z}$ between $90^{\circ}$ and $180^{\circ}$. If the ratio $\mathrm{Gr}_{\mathrm{v}} / \mathrm{Gr}$ is sufficiently high (larger than 2 in long 2D cavities), the roll can even change its orientation and become clockwise between two angles $\alpha_{1}$ and $\alpha_{2}$ at equal distance of $90^{\circ}$ and $180^{\circ}$, respectively $\left(90^{\circ}+\alpha_{0} / 2\right.$ and $180^{\circ}-\alpha_{0} / 2$, with $\alpha_{0}=\operatorname{asin}\left[2 /\left(\mathrm{Gr}_{\mathrm{v}} / \mathrm{Gr}\right)\right]$ in the model corresponding to long $2 \mathrm{D}$ cavities $)$. In our case with $\mathrm{Gr}_{\mathrm{v}} / \mathrm{Gr}=10$, our simulations in the cubic cavity give $\alpha_{0} / 2=11.9^{\circ}$, compared to $5.77^{\circ}$ according to the model. Such $x z$ vibrations are then particularly interesting for crystal growth applications as for vibration angles $\alpha_{x z}$ close to $45^{\circ}$, they can generate intense flows (up to a $255 \%$ intensification) which can provide good mixing and for vibrations angles close to the flow inversion limits $\alpha_{1}$ and $\alpha_{2}$, they can lead to weak flows (with a 93\% decrease) and rather diffusive conditions.

The influence of the $x y$ vibration is different as the vibration force now generates a roll in the $x y$ plane. The resulting flow is amplified compared to the buoyant flow, but its principal characteristic is that the main cell is shifted from the $x z$ plane towards the $x y$ plane, in a given direction for $\alpha_{x y}$ between $0^{\circ}$ and $90^{\circ}$ and symmetrically in the opposite direction for $\alpha_{x y}$ between $180^{\circ}$ and $90^{\circ}$ (the maximum shifts are obtained close to $45^{\circ}$ and $135^{\circ}$, respectively). Such flows have lost the main symmetries of the buoyant flow and only kept the central symmetry. Concerning crystal growth applications, these flows are interesting for mixing, with their increased intensity and the possibility of changing the orientation of the main roll.

Finally, the $y z$ vibration (in a plane parallel to the heated side walls) has clearly the weakest influence on the buoyant flow. Such vibrations, which keep the symmetries of the buoyant flow, will only slightly amplify this buoyant flow. The resulting flow still corresponds to a main cell in the $x z$ plane, but this cell is modified with different three-dimensional effects which will affect the global circulation of the fluid. In particular, the $v$ transverse velocity, which is weak in the buoyant flow, is clearly amplified for $\alpha_{y z}$ close to $0^{\circ}$. Note that the influence of the $y z$ vibration is the same for $\alpha_{y z}$ below or above $90^{\circ}\left(90^{\circ}-\alpha\right.$ or $\left.90^{\circ}+\alpha\right)$. Concerning crystal growth applications, the $y z$ vibrations seem to be the less interesting as the amplification of the flow they provide remains weak and cannot really improve mixing, despite increased three-dimensional effects.

\section{ACKNOWLEDGMENTS}

This collaborative work was supported by the PHC Maghreb Partnership Program No. 36951NG. A grant of the PROFAS B+ program given to A.M. is also gratefully acknowledged.

[1] G. Müller, Convection and inhomogeneities in crystal growth from the melt, in Crystal Growth from the Melt. Crystals (Growth, Properties, and Applications) (Springer-Verlag, Berlin, Heidelberg, 1988), Vol. 12, pp. 1-138.

[2] N. Ma and J. S. Walker, Magnetic damping of buoyant convection during semiconductor crystal growth in microgravity: Spikes of residual acceleration, Phys. Fluids 9, 1182 (1997).

[3] M. R. Foster, Asymptotic analysis of a three-dimensional Bridgman furnace at large Rayleigh number, Phys. Fluids 11, 1827 (1999).

[4] S. Tanveer, Convection effects on radial segregation and crystal-melt interface in vertical Bridgman growth, Phys. Fluids 6, 2270 (1994). 
[5] S. Kaddeche, H. Ben Hadid, and D. Henry, Macrosegregation and convection in the horizontal Bridgman configuration. I. Dilute alloys, J. Cryst. Growth 135, 341 (1994).

[6] S. Kaddeche, H. Ben Hadid, and D. Henry, Macrosegregation and convection in the horizontal Bridgman configuration. II. Concentrated alloys, J. Cryst. Growth 141, 279 (1994).

[7] G. Z. Gershuni and D. V. Lyubimov, Thermal Vibrational Convection (Wiley, New York, 1998).

[8] V. Shevtsova, D. Melnikov, J. C. Legros, Y. Yan, Z. Saghir, T. Lyubimova, G. Sedelnikov, and B. Roux, Influence of vibrations on thermodiffusion in binary mixture: A benchmark of numerical solutions, Phys. Fluids 19, 017111 (2007).

[9] S. M. Zen'kovskaya and I. B. Simonenko, Influence of high-frequency vibration on the onset of convection, Izv. Akad. Nauk SSSR, Mekh. Zhidk. Gaza 5, 51 (1966).

[10] A. V. Straube, D. V. Lyubimov, and S. V. Shklyaev, Averaged dynamics of two-phase media in a vibration field, Phys. Fluids 18, 053303 (2006).

[11] M. Lappa, Thermal Convection: Patterns, Evolution and Stability (John Wiley \& Sons, New York, 2010).

[12] T. J. O'Hern, B. Shelden, J. R. Torczynski, and L. A. Romero, Bubble oscillations and motion under vibration, Phys. Fluids 24, 091108 (2012).

[13] M. Lappa, On the variety of particle accumulation structures under the effect of g-jitters, J. Fluid Mech. 726, 160 (2013).

[14] C. Ferrera, M. A. Herrada, J. M. Montanero, M. Torregrosa, and V. Shevtsova, Dynamical response of liquid bridges to a step change in the mass force magnitude, Phys. Fluids 26, 012108 (2014).

[15] M. Lappa, Control of convection patterning and intensity in shallow cavities by harmonic vibrations, Micrograv. Sci. Technol. 28, 29 (2016).

[16] M. Lappa, Patterning behavior of gravitationally modulated supercritical Marangoni flow in liquid layers, Phys. Rev. E 93, 053107 (2016).

[17] M. Gaponenko, A. Mialdun, and V. Shevtsova, Pattern selection in miscible liquids under periodic excitation in microgravity: Effect of interface width, Phys. Fluids 30, 062103 (2018).

[18] S. Bouarab, F. Mokhtari, S. Kaddeche, D. Henry, V. Botton, and A. Medelfef, Theoretical and numerical study on high frequency vibrational convection: influence of the vibration direction on the flow structure, Phys. Fluids 31, 043605 (2019).

[19] S. Bouarab, F. Mokhtari, S. Kaddeche, D. Henry, V. Botton, and A. Medelfef, Effect of high frequency vibrations on PV silicon purification, J. Cryst. Growth 529, 125298 (2020).

[20] S. Biringen and L. J. Peltier, Numerical simulation of 3-D Bénard convection with gravitational modulation, Phys. Fluids A 2, 754 (1990).

[21] R. Savino, R. Monti, and M. Piccirillo, Thermovibrational convection in a fluid cell, Comput. Fluids 27, 923 (1998).

[22] A. Farooq and G. M. Homsy, Streaming flows due to g-jitter-induced natural convection, J. Fluid Mech. 271, 351 (1994).

[23] V. Shevtsova, I. Ryzhkov, D. E. Melnikov, Y. A. Gaponenko, and A. Mialdun, Experimental and theoretical study of vibration-induced thermal convection in low gravity, J. Fluid Mech. 648, 53 (2010).

[24] N. L. Trefethen, Spectral methods in Matlab (SIAM, Philadelphia, 2000).

[25] H. Johnston and J.-G. Liu, Accurate, stable and efficient Navier-Stokes solvers based on explicit treatment of the pressure term, J. Comput. Phys. 199, 221 (2004).

[26] H. Johnston, C. Wang, and J.-G. Liu, A local pressure boundary condition spectral collocation scheme for the three-dimensional Navier-Stokes equations, J. Sci. Comput. 60, 612 (2014).

[27] A. Medelfef, D. Henry, A. Bouabdallah, and S. Kaddeche, Bifurcations from steady to quasi-periodic flows in a laterally heated cavity filled with low Prandtl number fluids, J. Fluid Mech. 861, 223 (2019). 